\title{
A cooperative negotiation embedded NSGA-II for solving an integrated product family and supply chain design problem with remanufacturing consideration
}

\author{
Zhiqiao WU ${ }^{1,2}$, C.K. Kwong ${ }^{1}$, Ridvan Aydin ${ }^{1}$ and Jiafu Tang ${ }^{2}$ \\ ${ }^{1}$ Department of Industrial and Systems Engineering, The Hong Kong Polytechnic University, \\ Hung Hom, Kowloon, Hong Kong, P. R. China \\ ${ }^{2}$ College of Management Science \& Engineering, Dongbei University of Finance and \\ Economics, Dalian 116025, PR China
}

\begin{abstract}
Product family design is a popular approach adopted by manufacturers to increase their product varieties in order to satisfy the needs of various markets. In recent years, because of increasing environmental concerns in societies and strict regulations of environmental protection, quite a number of manufacturers adopted remanufacturing strategy in their product development in response to the challenges. Remanufacturing of used products unavoidably involves a closed-loop supply chain system. To achieve the best outcomes, the supply chain design should be considered in product family design process. In this research, a multiobjective optimization model of integrated product family and closed loop supply chain design is formulated based on a cooperative game model for minimizing manufacturer's total cost and maximize suppliers' total payoffs. Since the optimization problem could be a largescale one and involves mixed continuous-discrete variables, a new version of nondominated sorting genetic algorithm-II (NSGA-II), namely cooperative negotiation embedded NSGA-II (NSGA-CO), is proposed to solve the optimization model. Simulation tests are conducted to validate the effectiveness of the proposed NSGA-CO. The test results indicate that the proposed NSGA-CO outperforms NSGA-II in solving various scale of multi-objective optimization problems in terms of convergence. With the formulated optimization model and the proposed NSGA-CO, a case study of integrated product family and supply chain design is conducted to investigate the effects of environmental penalty, quantity of demand and marginal cost of remanufacturing on used product return rate, manufacturers' and suppliers' profits and joint payoff.
\end{abstract}

Keywords: Product family design; Negotiation; Remanufacturing; Multi-objective optimization; NSGA-II. 


\section{Introduction}

To satisfy the needs of various markets, many manufacturers have adopted product family design (PFD) to increase their product varieties in which different versions of product modules are used to create product variants that satisfy the needs of various markets. In recent years, manufacturers have been facing the challenges of increasing social concerns on environmental friendliness and stringent environmental regulations imposed by different governments. For example, the Directive 2002/96/EC (European Parliament and The Council) of WEEE-directive was imposed on all EU members to develop legislation based on Extended Producer Responsibility (EPR). EPR places the responsibility for taking back and recovering returned products on the original equipment manufacturers (OEMs) [1]. Thus, more and more manufacturers adopted remanufacturing strategy in their product development to respond to the challenges which unavoidably involve design of closed loop supply chain networks [2]. Therefore, supply chain design needs to be considered in product family design process in order to obtain the best outcomes.

Numerous studies were conducted about the integrated product family and supply chain design. Some of them mainly addressed the integration of supplier selection with platform product design. Ho et al. [3] and Hussien and Abdelsalam [4] reviewed the literature of simultaneous consideration of product family design and supplier selection. Gupta and Krishnan [5] formulated an integrated component and supplier selection problem for a product family as an integer programming model. A model of integrating supplier selection with product family design was formulated and solved by a linear programming embedded genetic algorithm [6]. A nonlinear optimization model under multinomial logit consumer choice rule for the integrated problem was also formulated [7]. Balakrishnan and Chakravarty [8] studied the integration of product variants and supplier selection as a profit-driven decision making problem. Huang et al. [9] and Zhang et al. [10] studied simultaneous configuration of platform products and supply chains under two scenarios: non-cooperative and cooperative suppliers respectively. Deng et al. [11] formulated an integrated product line design and supplier selection problem using a multi-objective optimization paradigm. Yang et al. [12] proposed a bi-level nested genetic algorithm to solve joint configuration of product family and supply chain problems. Baud-Lavigne et al. [13] later proposed a mixed integer linear programming model for simultaneous product family and supply chain design.

Quite a few studies of integrated product family and supply chain design with consideration of remanufacturing were attempted. Xu et al. [14] firstly formulated a re-use 
methodology of PFD to evaluate product performance based on component re-use. Ferrer and Swaminathan [15] studied the problem of producing new products in the first period and using returned cores to produce remanufactured products (regard as product portfolio) in future periods. Wang et al. [16] developed a hybrid manufacturing/remanufacturing model to examine the optimal policies on the manufacturing and remanufacturing returned products. Metta and Badurdeen [17] studied the modeling issues and challenges of integrated product and supply chain design with consideration of reuse, remanufacturing and recycling. Wu et al. [18] addressed a joint decision of PFD and remanufacturing with consideration of supplier selection. He [19] examined centralized and decentralized closed-loop supply chain network models to determine the optimal return price of used products and the remanufacturing decisions. Based on the predefined requirements of product improvement and component attributes, Tao and $\mathrm{Yu}$ [20] formulated product line-ups and modeled changing plan to minimize the total environmental impacts. Subramanian et al. [2] studied how remanufacturing could reverse the OEM's commonality decision that was based on the manufacturing and sales of new products. Aydin et al. [21] studied product line design with consideration of remanufactured products by modeling coordination of the closed-loop supply chain parties. In previous studies, various approaches and algorithms were developed / employed to solve integrated product and supply chain design problems that involve one objective such as heuristic procedures [5, 13], genetic algorithms [6, 9, 20] and Lagrangian relaxation based approach [22]. As some integrated problems studied in previous research involve multi-objectives, various approaches and algorithms were employed to solve the multi-objective optimization problems such as bi-level nested genetic algorithm [12], nondominated sorting genetic algorithm-II [11, 18, 21] and three-phase heuristic algorithm [23].

Two main characteristics of the optimization models for integrated product family and supply chain design with consideration of remanufacturing can be seen; (1) Remanufacturing decisions involve a number of tradeoffs, competition and negotiation such as competition and negotiation between a manufacturer and retailers [24] and between a manufacturer and remanufacturers [25], as well as the tradeoff between the purchase of brand new components and remanufactured components [26]; (2) The optimization problems are quite often multiobjective ones with commonly involving both continuous variables such as discount prices and return rate and discrete variables such as product configuration. One common technique of solving multi-objective optimization problems is to minimize the positively weighted convex sum of objectives. However, the method has two drawbacks. First, in most cases, all 
the solutions find to be clustered in certain parts of the Pareto set with no point in the interested "middle part" of the set. Thus, few insights can be provided to the shape of the trade-off curve. Second, the solutions obtained depend greatly on the weights assigned by decision-makers [23]. An alternative solving technique is evolutionary algorithms, which are capable of evolving a set of Pareto optimal points (efficient solution) simultaneously and may exploit similarities among regions in a search space. However, the decision variables involved in the integrated problems may include continuous variables. Although a hybrid chromosome structure supporting both continuous and discrete variables can be adopted, the optimal setting of continuous variables is difficult to obtain using genetic search alone [6, 27].

The integrated product family and supply chain design problem with remanufacturing consideration quite often involves multiple objectives. It can be noted that Nondominated sorting genetic algorithm-II (NSGA II) was widely employed to solve the similar integrated problems in previous studies [11, 18, 21, 28]. However, NSGA-II is not capable of solving the optimization problems that involves continuous decision variables as non-dominated solutions are difficult to be obtained by using meta-heuristic search alone. Thus, in this research, a new version of NSGA-II, namely cooperative negotiation embedded NSGA-II (NSGA-CO), is proposed to solve the integrated problem. Based on the formulated optimization model and NSGA-CO, the effects of environmental penalty, quantity of demand and the marginal cost of remanufacturing on used product return rate, manufacturers' and suppliers' profits and joint payoff can be studied.

The rest of the paper is organized as follows. Section 2 presents our proposed methodology for solving an integrated product family and supply chain design problem. Section 3 describes the formulation of a multi-objective optimization model for integrated product family and supply chain design with consideration of remanufacturing. The NSGA$\mathrm{CO}$ for solving the integrated problem is described in section 4. Section 5 presents the simulation tests and the results for validating the effectiveness of NSGA-CO. A case study of integrated product family and supply chain design for printing calculators and the effects of environmental penalty, quantity of demand and the marginal cost of remanufacturing on used product return rate, manufacturers' and suppliers' profits and joint payoff are presented in Section 6. Finally, conclusions are provided in Section 7. 


\section{A proposed methodology for solving the problem of integrated product family and supply chain design with remanufacturing}

A decentralized closed-loop supply chain model for PFD with remanufacturing as shown in Fig. 1 is considered in this research. In the closed loop supply chain network, the manufacturer undertakes the collection of used products or subcontract the collection activity to retailers, and decides the product return rate. After the collection, the used products are decentralized to her multiple cooperative suppliers for remanufacturing. The suppliers can provide remanufactured components to the manufacturer with lower prices compared with those of the new components. This may attract the manufacturer to increase the product return rate if the prices of remanufactured components are attractive. On the other hand, the suppliers have to perform a tradeoff between ordering new materials for producing components and collecting used components for remanufacture. The manufacturer needs to design their product variants with consideration of the use of new and/or remanufactured components. Suppliers may formulate discount price strategies for enabling the manufacturer to increase the product return rate.

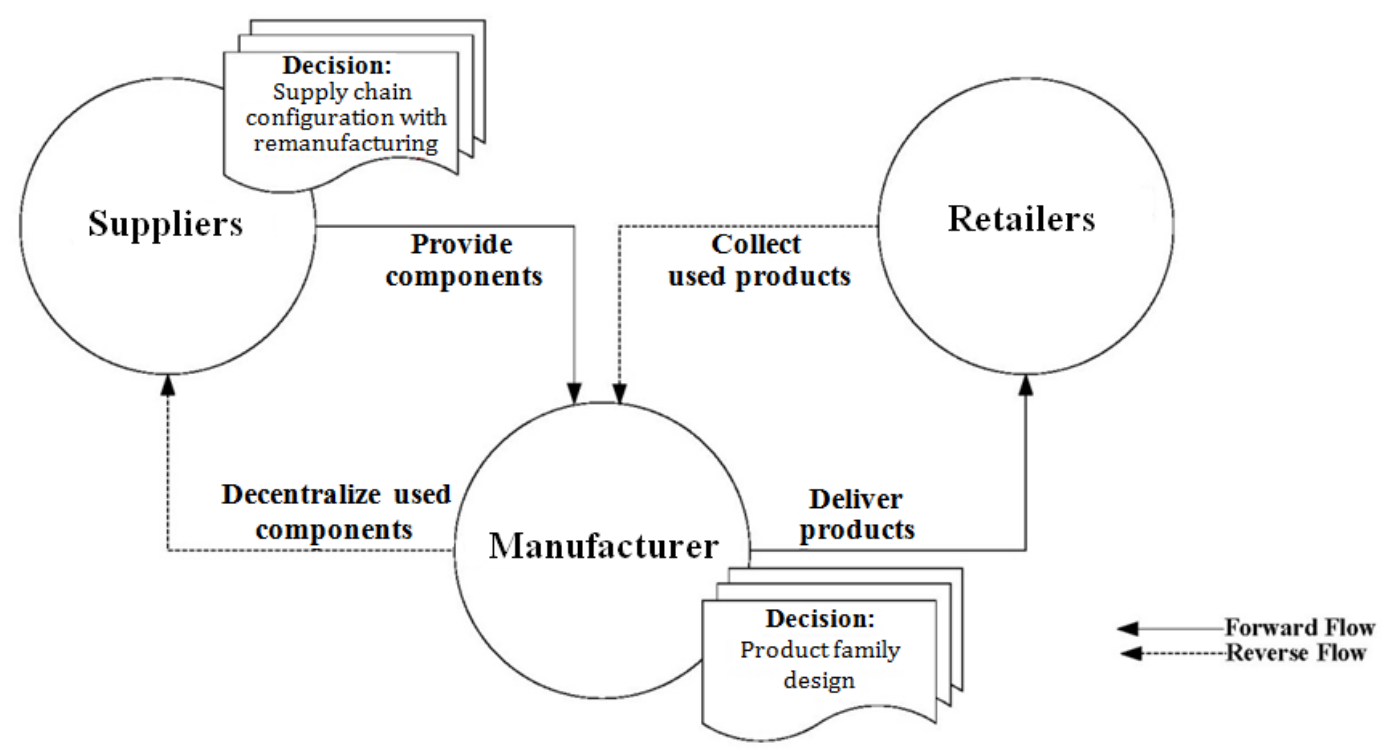

Fig. 1. The closed-loop supply chain with remanufacturing.

In the proposed methodology, a multi-objective optimization model with the objectives of minimizing the manufacturer total cost and maximizing the suppliers' payoff is formulated using a game model. The game model to be adopted is a cooperative one where two players bargain with complete information about the game. Therefore, each player has the information of the payoff or profit for himself and their co-players in every possible 
transaction. By solving the optimization model, the Pareto-optimal equilibrium for product configuration, supplier selection, product return rate and discounted return prices can be obtained. With the optimization model, computational experiments can be conducted to investigate the effects of environmental penalty, quantity of demand and the marginal cost of remanufacturing on used product return rate, manufacturers' and suppliers' profits and joint payoff.

The formulated multi-objective optimization model quite often involves both discrete and continuous decision variables. Since the game model involves multiple players, determination of the Pareto-optimal equilibrium in the mixed strategies involves solving a system of high order multivariate polynomial equations which leads to a numerical challenge to obtain an explicit solution. The high complexity of the optimization problem due to the large number of players and the temporal constraints of generating optimal solutions hinders the adoption of conventional techniques to solve the bargaining models [29]. An alternative solving technique is multi-objective evolutionary algorithms which were commonly adopted to solve multi-objective optimization problems. Various types of multi-objective evolutionary algorithms were developed such as strength Pareto evolutionary algorithm, Pareto Archive Evolution Strategy and NSAG-II. Among the various types, Deb et al. [30] found that NSGA-II was able to maintain better spread of solutions and had better convergence than other multi-objective evolution algorithms, such as Pareto-archived evolution strategy and strength-Pareto evolutionary algorithm. Coello [31] also conducted a study to benchmark various types of multi-objective evolutionary algorithms and found that NSGA-II was more computationally efficient than other multi-objective evolutionary algorithms. However, NSGA-II is not capable of solving the multi-objective optimization problems that involves continuous decision variables as non-dominated solutions are difficult to be obtained by using meta-heuristic search alone. In this paper, a new version of NSGA-II, namely cooperative negotiation embedded NSGA-II (NSGA-CO), is proposed to solve the multi-objective optimization problem that involve mixed discrete-continuous variables and game models with multi-players as well as would be highly complex and large-scale. In the proposed algorithm, heuristics are developed to determine continuous variables setting (i.e. return rate and discounted pricing) for maximizing joint payoff of the manufacturers and cooperative suppliers by finding an egalitarian bargaining solution. In the following, formulation of a multi-objective optimization model for integrated product family and supply chain design with remanufacturing consideration and the proposed NSGA-CO are described. 


\section{Model formulation}

Suppose a manufacturer would like to design a family of new products to satisfy the diversified needs of various markets. A set of internal interfaces are defined for the products, so that for each interface, a number of components with similar functionality but different levels of performance can be fully replaceable. A product variant can be obtained by selecting one component for each interface. Following Gupta and Krishnan's notation [5], components with different levels of the same functionality can be defined as a set of the replaceable component set (RCS).

Assumption 1. In the scenario of a closed-loop supply chain system, the manufacturer undertakes the collection of used products. The used components are delivered to suppliers for remanufacturing.

Suppose that some suppliers have incorporated remanufacturing processes into their production systems so that they can restore used components functionally to their original or even better condition. Remanufactured components can be utilized to produce product variants in PFD. The manufacturer always plays the role of the leader and her suppliers act as the followers that can be modeled as a Stackelberg game in PFD. Major decision variables of the manufacturer in the optimization are the configurations of product variants and number of used products to be collected that involves the determination of product return rate. For the suppliers, major decision variables in the optimization are desired product return rate and the discount price of the components for a particular return rate. To facilitate the modeling, the following notations are used to define decision variables:

$x_{i j}$ : a binary variable; $x_{i j}=1$ if the $j$-th product variant in the $i$-th segment is chosen, and 0 otherwise;

$y_{j k l v}$ : a binary variable; $y_{j k l v}=1$ if the $l$-th component (provided by the $v$-th supplier) of the $k$-th RCS is selected for the $j$-th product variant, and 0 otherwise;

$e_{j k l v}$ : a continuous variable; $e_{j k l v} \in\left(c_{j k l v}^{0}, e_{j k l v}^{0}\right]$, and represents the price of the $l$-th component (provided by the $v$-th supplier) of the $k$-th RCS for the $j$-th product variant, where $e_{j k l v}^{0}$ is the $v$-th supplier's bidding price for the $l$-th component of the $k$-th RCS and $c_{j k l v}^{0}$ denotes the cost of manufacturing $l$-th component of the $k$-th RCS for the $v$-th supplier; $\tau_{j}$ : a continuous variable; $\tau_{j} \in(0,1)$, and denotes the return rate of the $j$-th used product variant. 


\subsection{Modeling customer preferences}

Conjoint analysis (CA) is a popular method used to understand customer preferences in consumer market research. CA assumes that an individual's overall utility for a product is the sum of part-worth utilities for each attribute level of the product [32]. Following the partworth utility model, the utility of a product variant can be expressed as a linear function of the part-worth utilities of components for the RCSs as follows:

$$
U_{i j}=\sum_{k=1}^{K} \sum_{l=1}^{L_{k}} \sum_{v=1}^{V} w_{j k} u_{i k l} y_{j k l v}
$$

where $U_{i j}$ is the utility of the $j$-th product variant in the $i$-th segment; $w_{j k}$ is the weight of the $j$-th product variant in the $k$-th RCS; and $u_{i k l}$ is the part-worth utility of the $l$-th component of the $k$-th RCS in the $i$-th segment. $K$ and $L_{k}$ represent the number of RCSs and number of components in the $k$-th $\operatorname{RCS}(k=1, \ldots, K)$, respectively; and $y_{j k l v}$ is a binary variable. $y_{j k l v}=1$ represents the $l$-th component (provided by the $v$-th supplier) of the $k$-th RCS is selected for the $j$-th product variant, and 0 otherwise.

Consumers of a market may have different preferences on product attributes. Thus, the market can be partitioned into a number of segments. First, a market survey needs to be conducted to understand consumer preferences on different product profiles. Based on the survey data, market segments can be identified using proper clustering techniques [33] and the size of each market segment can also be estimated [6]. The hurdle utility for the $n$-th customer in the $i$-th segment is denoted as $h_{i n}$ and $h_{i n}=U_{i n}+\varepsilon$, where $U_{i n}$ is the reservation utility of the $n$-th respondent in the $i$-th segment and $\varepsilon$ denotes the random error of the utility and is a small positive real number.

\subsection{Development of cost models}

The cost model for the manufacturer involves the cost of components purchased from suppliers, greening cost, and collection cost of used products. The total cost of producing a product by the manufacturer can be used to form an objective function that will be described later. The cost model for suppliers involves the cost of producing a new component, remanufacturing cost of a component, and the investment on remanufacturing.

Assumption 2. In this scenario of a closed-loop supply chain system, to characterize the diminishing return to investment, the cost structure of the investment can be formulated as 
$c_{j k l}^{L} \tau_{j}^{2}$, where $c_{j k l}^{L}$ is a scaling parameter of the l-th returned component of the $k$-th RCS for the $j$-th product variant [24].

Similar forms of functions have been widely used in the advertising response models of consumer retention and product awareness [34, 35].

\subsection{Formulation of an optimization model}

A multi-objective optimization model is formulated to solve the problem of integrated product family and supply chain design with remanufacturing consideration. Two objective functions are considered in the formulation. The first objective function is to minimize manufacturer's total cost of producing product variants as shown below.

$$
\sum_{j=1}^{J} \sum_{k=1}^{K} \sum_{l=1}^{L} \sum_{v=1}^{V}\left(d_{j k l} e_{j k l v} y_{j k l v}+\frac{B_{j}}{\tau_{j}}+A_{j} d_{j} \tau_{j}\right)
$$

where $d_{j k l}$ represents a linear demand of the $l$-th component of the $k$-th RCS for the $j$-th product variant [36]; $d_{j}$ is the demand of the $j$-th product variant; $B_{j}$ is the penalty cost of the $j$-th product variant which is equal to $d_{j} \cdot \varphi_{j}$ and $\varphi_{j}$ is the environmental penalty factor of the $j$ th product variant that relates to environmental (green) supply chain performance metric [37, 38]; and $A_{j}$ denotes the unit cost of collecting and handling the $j$-th returned product variant. The three terms inside the brackets of the Eq. (2) refer to the purchasing cost of components, greening cost, and collection cost of used products, respectively.

The second objective of the optimization model is to maximize suppliers' total profits from the sales of components to the manufacturer, which can be expressed as follows:

$$
\sum_{v=1}^{V} \pi_{v}=\sum_{j=1}^{J} \sum_{k=1}^{K} \sum_{l=1}^{L_{k}} \sum_{v=1}^{V} y_{j k l v}\left(d_{j k l}\left(e_{j k l}-c_{j k l}^{m}\right)+d_{j k l} \Delta_{j k l} \tau_{j}-c_{j k l}^{L} \tau_{j}^{2}\right)
$$

where $\pi_{v}$ is the profit of the $v$-th supplier; $\mathrm{V}$ is the total number of suppliers; $c_{j k l}^{m}$ is the unit cost of manufacturing the $l$-th new component of the $k$-th RCS for the $j$-th product variant; $c_{j k l}^{r}$ is the unit cost of remanufacturing the $l$-th returned component of the $k$-th RCS for the $j$ th product variant; $d_{j k l}\left(e_{j k l}-c_{j k l}^{m}\right)$ is the profit obtained from selling new components; $d_{i k l} \Delta_{j k l} \tau_{j}$ is the cost saving obtained from producing remanufactured components; and $c_{j k l}^{L} \tau_{j}^{2}$ is the supplier's investment on remanufacturing facilities which was formulated based on Savaskan's work [24]. 
In addition, the following constraints are required in the formulation of the optimization model.

$$
\begin{aligned}
& \sum_{i=1}^{I} \sum_{j=1}^{J} x_{i j} \leq H \\
& \sum_{k=1}^{K} \sum_{l=1}^{L_{k}} \sum_{v=1}^{V} w_{j k} u_{i k l} y_{j k l v} \geq h_{i} x_{i j}, \\
& \Delta_{j k l}=c_{j k l}^{m}-c_{j k l}^{r} \geq A_{j}, j=1,2, \ldots, J ; k=1,2, \ldots, K ; l=1,2, \ldots, L_{k}
\end{aligned}
$$

Constraints (4) ensures that the number of generated product variants is less than or equal to the pre-defined maximum number of product variants in a product family, $H$. Constraint (5) is introduced to ensure that $x=0$ when no constituted product has a utility higher than the respondent's reservation utility. Constraint (6) ensures that the unit cost savings is larger than or equal to the collecting and handling cost of a product.

\section{Cooperative negotiation embedded NSGA-II}

The model formulated in Section 3.3 is a multi-objective optimization one which involves both discrete and continuous variables as well as non-continuous objective function. Although deterministic optimization algorithms could be considered, they were found difficult to solve a problem with a non-continuous objective function [40]. As mentioned in Section 2, NSGA-II is one type of multi-objective evolutionary algorithms which was widely used to solve multi-objective optimization problems. However, the formulated optimization model involves both discrete and continuous variables that makes the optimal setting of continuous variables difficult to be determined using genetic search alone $[6,27]$. In this paper, a new version of NSGA-II, namely cooperative negotiation embedded NSGA-II (NSGA-CO), is proposed to solve the multi-objective optimization problem. The notion of nondominated sorting genetic algorithm (NSGA) was first suggested by Goldberg [41] and further studied by Srinivas and Deb [42] to solve multi-objective optimization problems. Deb et al. [30] proposed the improved multi-objective optimization solution algorithm, NSGA-II, by reducing computational complexity and providing a fast and effective constraint-handling strategy which makes it one of the most efficient multi-objective evolutionary algorithms. A flowchart of NSGA-II algorithm is shown in Appendix A. With the use of NSCA-CO in solving the optimization problem, two layers of variables of the formulated optimization model needs to be defined. The first layer involves the binary decision variables representing the feasible combinations of product configuration and supplier selection. The second layer involves the continuous decision variables, return rate and discounted return price, for each combination. In the proposed NSGA-CO, NSGA-II is used to perform the stochastic search 
for the binary variables in the first layer. It is regarded as integrating supplier selection in optimal product family design without considering remanufacturing (i.e. $\tau_{j}$ is set as a small positive real number $\varepsilon$ and the component price is set as the supplier's bidding price $e_{j k l v}^{0}$ ). The continuous variables are considered in a dynamic cooperative game. A heuristic approach is employed to determine the continuous variables setting by finding an egalitarian bargaining solution of the dynamic cooperative game, by which the return rate and discounted return price can be determined to maximize the joint payoff of the players. The fitness value of current population is updated by using a non-dominated sorting. Thus, in principle, Pareto optimal solution can be obtained by searching all feasible combinations iteratively. Fig. 2 shows the overall procedure of NSGA-CO.

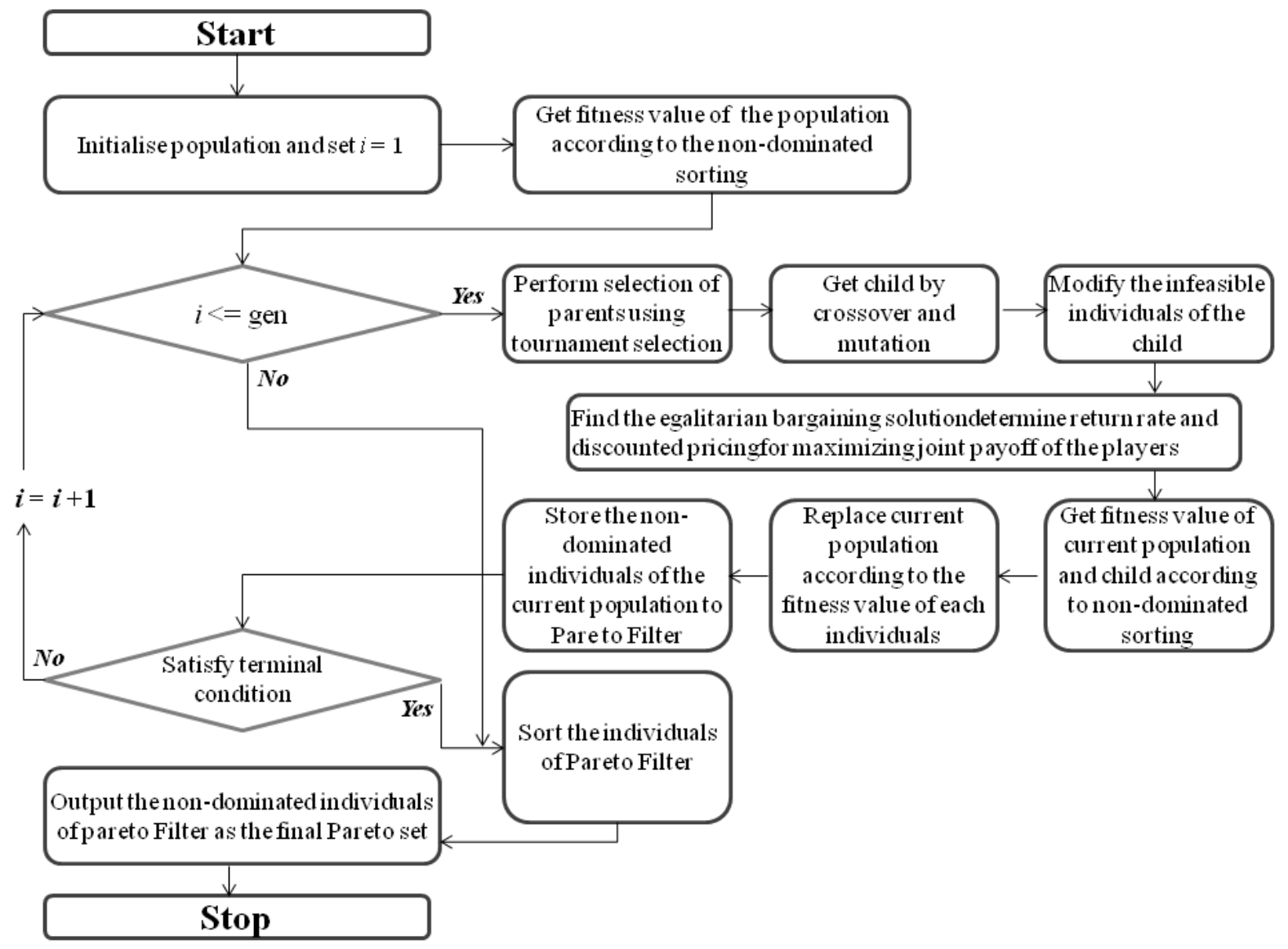

Fig. 2. Overall procedures of the NSGA-CO.

In NSGA-CO, considering that the number of continuous variables is highly related to the discrete variables, the discrete variables is used in the heuristic search, and a cooperative dynamic game routine is adopted to determine the optimal setting of continuous variables for the given setting of discrete variables. Since the discrete variables $x_{i j}$ and $y_{j k v}$ are correlated, 
only the representation of $y_{j k v}$ is used in the chromosome coding of NSGA-CO. Therefore, chromosome coding only involves the product configuration, which comprises $I$ sub-sections. A sub-section contains $K$ units, and the integer value in the $k$-th unit represents that the $l$-th component is selected for the RCS. A chromosome consists of two sections, return rate section and product configuration section. A return rate section has $I$ genes and the continuous value in the $i^{\text {th }}$ gene represents that the return rate of the $j^{\text {th }}$ used product variant. Fig. 3 shows an example of an encoded chromosome. The example involves two product variants and four RCSs. According to the coding of return rate section, the first product variant and the second product variant return rate are set as 0.3 and 0.2 , respectively. According to the coding of product configuration sub-section 1, the first product variant has the following configuration information; 'The first RCS uses the first component with price $\$ 4.2$; the second RCS uses the second component with price \$3.1; the third RCS uses the third component with price $\$ 1.6$ and the fourth RCS uses the first component with price $\$ 2.2^{\prime}$. Sub-section 2 has a similar encoding mechanism.

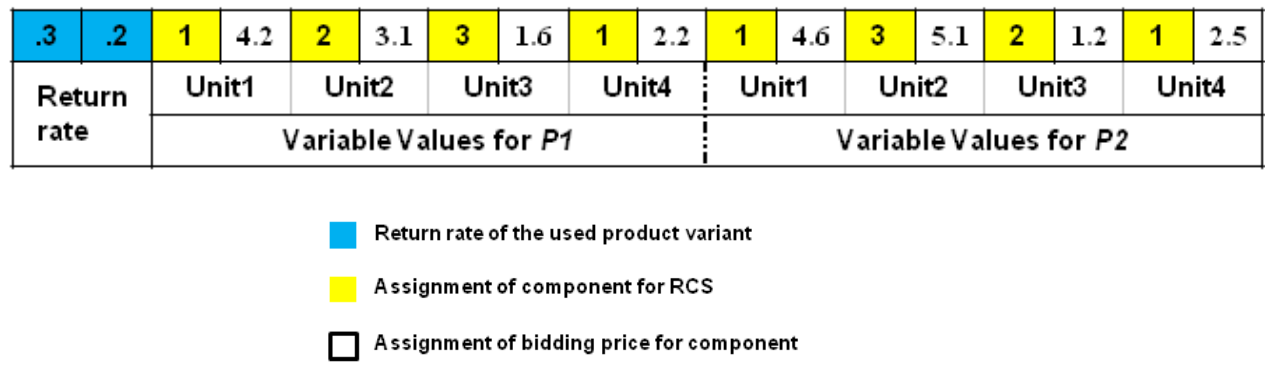

Fig. 3. An example of an encoded chromosome.

Selection of genetic operator, repairing strategy, termination condition, and Pareto filter of NSGA-CO are similar to those of NSGA-II.

\subsection{Cooperative negotiation heuristics}

An individual chromosome represents a possible combination of products and suppliers according to the representation scheme. The binary variables can be obtained via a decoding process which can help satisfy the constraints (4), (5) and (6), where the only continuous decision variables, discounted return price and return rate, need to be determined. This section describes a cooperative negotiation heuristic approach to determine the value setting of the continuous decision variables. 
Proposition 1. The form of the optimal contract $x=\left(\tau_{j}^{x}, G_{v}^{x}\right)$, which ensures that the manufacturer undertakes the return rate $\tau_{j}^{x}$ and charges the optimal discount price $G_{v}^{x}$.

To ensure the manufacturer and suppliers accept the contract, an egalitarian bargaining solution suggests that the players will maximize their utilities subject to the restriction that they all gain "equally" in the given situation [43]. This research employs a discount pricing strategy to derive the egalitarian bargaining solution. First, assume that no discount schedule is initially offered by supplier $v$, i.e. $e_{j k l}=e_{j k l}^{0}$, and at the same time, the manufacturer determines her initial return rate $\tau_{j}^{0}$ of used products collected from customers or retailers. Let $\Pi_{j v}(0)$ denote the manufacturer's purchasing cost from supplier $v$ without discounts. Eq. (7) can be obtained as follows.

$$
\Pi_{v}(0)=\Sigma_{j}^{J}\left(\sum_{k}^{K} \sum_{l}^{L_{k}} d_{j k} e_{j k l}^{0} y_{j k l}+\frac{B_{j}}{\tau_{i}^{0}}+A_{j} d_{j} \tau_{j}^{0}\right)
$$

Let $G_{v}^{x}$ denote the purchasing cost of $d_{j k l}$ units of components supplied by $v$. Therefore, the average unit price for an order of $d_{j k l}$ units is $G_{v}^{x} / d_{j k l}$.

$$
G_{v}^{x}=\Sigma_{j}^{J} \sum_{k}^{K} \Sigma_{l}^{L_{k}} d_{j k l} e_{j k l}^{x} y_{j k l}
$$

Now, supplier $v$ requests the manufacturer to increase her return rate to $\tau_{j}^{x}\left(>\tau_{j}^{\circ}\right)$ by offering a discount schedule $\mathrm{x}=\left(\tau_{j}^{x}, G_{v}^{x}\right)$ in return. The manufacturer's total cost can be written as follows:

$$
\Pi_{v}(x)=G_{v}^{x}+\sum_{j}^{J} \frac{B_{j}^{\tilde{j}}}{\tau_{j}^{x}}+\sum_{j}^{J} A_{j} d_{j} \tau_{j}^{x}
$$

Subtracting Eq. (7) from Eq. (9) and setting $\Delta \Pi_{v}(x)=\Pi_{v}(0)-\Pi_{v}(x)$, where $\Delta \Pi_{v}(\mathrm{x})$ is a decrease of the manufacture's sourcing cost, Eq. (10) is obtained.

$$
\Delta \Pi_{v}(x)=G_{v}^{0}-G_{v}^{x}+\sum_{j}^{J} B_{j}\left(\frac{1}{\tau_{i}^{0}}-\frac{1}{\tau_{i}^{x}}\right)+\sum_{j}^{J} A_{j} d_{j}\left(\tau_{j}^{0}-\tau_{j}^{x}\right)
$$

Similarly, if the manufacturer purchases components at the price without any discount, total profit of supplier $v$ can be obtained as follows:

$$
\pi_{v}(0)=\Sigma_{j}^{J} \sum_{k}^{K} \sum_{l}^{L_{k}} d_{j k l}\left(e_{j k l}^{0}-c_{j k l}^{m}\right)+\sum_{j}^{J} \sum_{k}^{K} \sum_{l}^{L_{k}} d_{j k l} \Delta_{j k l} \tau_{j}^{0}-\Sigma_{j}^{J} \sum_{k}^{K} \Sigma_{l}^{L_{k}} c_{j k}^{L}\left(\tau_{j}^{0}\right)^{2}
$$

When the supplier $v$ offers a discount schedule $=\left(\tau_{j}^{x}, G_{v}^{x}\right)$, then its total profit $\pi_{v}(x)$ can be obtained as follows:

$$
\pi_{v}(x)=\sum_{j}^{J} \sum_{k}^{K} \sum_{l}^{L_{k}} d_{j k l}\left(e_{j k l}^{x}-c_{j k}^{m}\right)+\sum_{j}^{J} \sum_{k}^{K} \sum_{l}^{L_{k}} d_{j k l} \Delta_{j k l} \tau_{j}^{x}-\sum_{j}^{J} \sum_{k}^{K} \sum_{l}^{L_{k}} c_{j k l}^{L}\left(\tau_{j}^{x}\right)^{2}
$$

Let $\Delta \pi_{v}(x)=\pi_{v}(x)-\pi_{v}(0)$, where $\Delta \pi_{v}(x)$ is an increase in the supplier's profit due to the discount schedule $x$. Subtracting Eq. (12) from Eq. (11), Eq. (13) is obtained. 


$$
\Delta \pi_{v}(x)=G_{v}^{x}-G_{v}^{0}+\sum_{j}^{J} \sum_{k}^{K} \sum_{l}^{L_{k}} d_{j k l} \Delta_{j k l}\left(\tau_{j}^{x}-\tau_{j}^{0}\right)-\Sigma_{j}^{J} \sum_{k}^{K} \sum_{l}^{L_{k}} c_{j k l}^{L}\left(\left(\tau_{j}^{x}\right)^{2}-\left(\tau_{j}^{0}\right)^{2}\right)
$$

In order to reduce the mathematical complexity for finding an egalitarian bargaining solution where the manufacturer and suppliers all gain equally, theoretical analysis is performed with the above dynamic game.

From Eqs. (10) and (13), we obtain:

$$
\begin{gathered}
G_{v}^{x}=G_{v}^{0}-\Delta \Pi_{j v}+\sum_{j}^{J} B_{j}\left(\frac{1}{\tau_{i}^{0}}-\frac{1}{\tau_{i}^{x}}\right)+\sum_{j}^{J} A_{j} d_{j}\left(\tau_{j}^{0}-\tau_{j}^{x}\right) \\
G_{v}^{x}=\Delta \pi_{v}+G_{v}^{0}-\Sigma_{j}^{J} \sum_{k}^{K} \Sigma_{l}^{L_{k}} d_{j k l} \Delta_{j k l}\left(\tau_{j}^{x}-\tau_{j}^{0}\right)+\Sigma_{j}^{J} \Sigma_{k}^{K} \Sigma_{l}^{L_{k}} c_{j k l}^{L}\left(\left(\tau_{j}^{x}\right)^{2}-\left(\tau_{j}^{0}\right)^{2}\right)
\end{gathered}
$$

From Eq. (14), we can obtain:

$$
\begin{aligned}
& \frac{d G_{v}^{x}}{d \tau_{j}^{x}}=\sum_{j}^{J}-A_{j} d_{j}+\sum_{j}^{J} B_{j} /\left(\tau_{j}^{x}\right)^{2} \\
& \text { and } \frac{d^{2} G_{v}^{x}}{d\left(\tau_{j}^{x}\right)^{2}}=\sum_{j}^{J}-2 B_{j} /\left(\tau_{j}^{x}\right)^{3}
\end{aligned}
$$

From Eq. (15), we obtain

$$
\begin{gathered}
\frac{d G_{v}^{w}}{d \tau_{j}^{x}}=-\sum_{j}^{J} \sum_{k}^{K} \sum_{l}^{L_{k}} d_{j k l} \Delta_{j k l}+2 \sum_{j}^{J} \sum_{k}^{K} \sum_{l}^{L_{k}} c_{j k l}^{L} \tau_{j}^{x} \\
\text { and } \frac{d^{2} G_{w}^{w}}{d\left(\tau_{j}^{x}\right)^{2}}=2 \sum_{j}^{J} \sum_{k}^{K} \Sigma_{l}^{L_{k}} c_{j k l}^{L}
\end{gathered}
$$

Eq. (14) describes $G_{v}^{x}$ as a concave function of $\tau_{j}^{x}$ because the value of Eq. (17) is negative, and Eq. (15) describes $G_{v}^{x}$ as a convex function of $\tau_{j}^{x}$ because the value of Eq. (19) is positive. Given the values of $\Delta \Pi_{v}(\mathrm{x})$ and $\Delta \pi_{v}(x)$, Eqs. (14) and (15) specify a concave and a convex curve in the $\left(G_{v}^{x}, \tau_{j}^{x}\right)$ space, respectively. To illustrate how the feasible set of discounts is found, two case scenarios are described as follows.

\subsubsection{Case Scenario 1}

Considering the following situation of a discount schedule of remanufacturing with one manufacturer and one supplier (A). First, no discount schedule is initially offered by supplier (A), and $e_{j k l}^{0}$ is set as $\$ 1450$, and at the same time, the manufacturer sets her return rate $\tau_{j}^{0}$ as 0.1 . The payment $A$ is set as $\$ 10$, which is given to a consumer who returns a used product. The penalty amount of $B_{j}$ is $\$ 20$. The demand $d$ of the product variant is 50 (Unit). The scaling parameter $c^{L}$ is 850 . The unit cost $c^{m}$ of manufacturing a new product is $\$ 250$, and the unit cost $c^{r}$ of remanufacturing a returned product is $\$ 215$. Based on the Eqs. (14) and (15), we can obtain a concave and a convex curve in the $\left(G_{v}^{x}, \tau_{j}^{x}\right)$ space as shown in Fig. 4. 


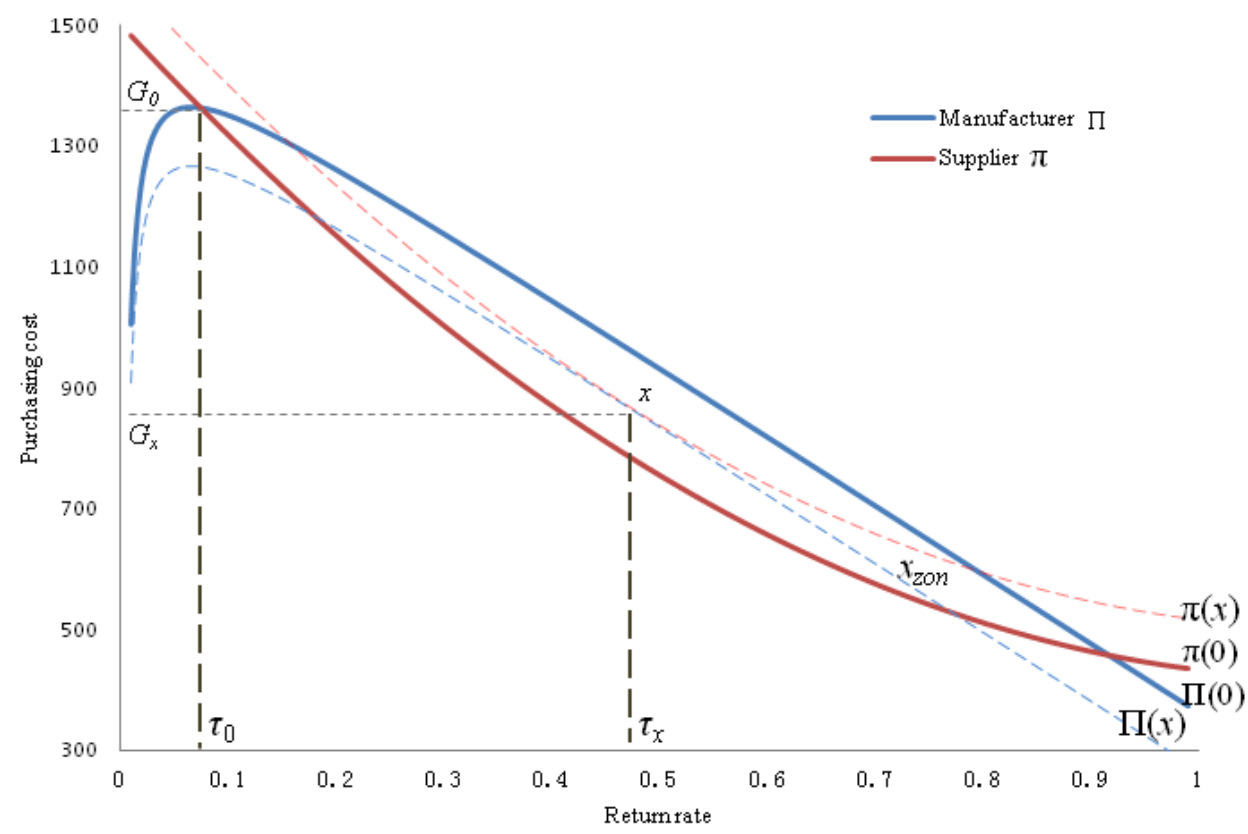

Fig. 4. A case of one manufacturer and one supplier (A).

When $\Delta \Pi_{v}(x)=\Delta \pi_{v}(x)=0$, the concave curve $\Delta \Pi_{v}(x)$ and the convex curve $\Delta \pi_{v}(x)$ intersect at point $\left(G_{v}^{0}, \tau_{j}^{0}\right)$. This corresponds to the condition that no discount schedule is offered by supplier (A). Referring to Fig. 4, a feasible discount always can be set by satisfying the conditions $\Delta \Pi_{v}(\mathrm{x}) \geq 0$ and $\Delta \pi_{v}(x) \geq 0$. We use $X_{\text {zon }}=\left\{\left(G_{v}^{x}, \tau_{j}^{x}\right) \mid \Delta \Pi_{v}(\mathrm{x}) \geq 0, \Delta \pi_{v}(x) \geq 0\right\}$ to denote the set within the boundary of curve $\Pi_{v}(0)$ and curve $\pi_{v}(0)$ in Fig. $4 . X_{z o n}$ is the feasible set of discounts. Therefore, the manufacturer and the supplier may bargain for a discount in $X_{z o n}$. One possible outcome of bargaining among a manufacturer and suppliers is to obtain the Pareto efficient of discounts as non dominated solution.

The values of $G_{v}^{\max }$ and $G_{v}^{\min }$ can be obtained by setting $\tau_{j}^{x}=\tau_{j}^{*}$ in curve $\Pi_{j v}(x)$ and curve $\pi_{j v}(x)$, respectively:

$$
\begin{gathered}
G_{v}^{\max }=G_{v}^{0}+\sum_{j}^{J} B_{j}\left(\frac{1}{\tau_{i}^{0}}-\frac{1}{\tau_{j}^{x}}\right)+\sum_{j}^{J} A_{j} d_{j}\left(\tau_{j}^{0}-\tau_{j}^{x}\right) \\
G_{v}^{\min }=G_{v}^{0}+\sum_{j}^{J} \sum_{k}^{K} \sum_{l}^{L_{k}} d_{j k l} \Delta_{j k l}\left(\tau_{j}^{x}-\tau_{j}^{0}\right)-\sum_{j}^{J} \sum_{k}^{K} \sum_{l}^{L_{k}} c_{j k l}^{L}\left(\left(\tau_{j}^{x}\right)^{2}-\left(\tau_{j}^{0}\right)^{2}\right)
\end{gathered}
$$

The optimal solution of discount schedule, denoted by $z=\left(G_{v}^{x}, \tau_{j}^{x}\right)$, is the point where the curves $\Pi_{v}(z)$ and $\pi_{v}(z)$ intersect for any supplier. Then we have:

$$
\begin{aligned}
& \Delta \Pi_{v}(z)=\Pi_{v}(0)-\Pi_{v}(z)=G_{v}^{\max }-G_{v}^{*} \\
& \Delta \pi_{v}(z)=\pi_{v}(z)-\pi_{v}(0)=G_{v}^{*}-G_{v}^{\min }
\end{aligned}
$$


Considering $\Delta \Pi_{v}(z)$ and $\Delta \pi_{v}(z)$, the objective function as shown below is to optimize all players' joint payoff in the cooperative game:

$$
\text { Payoff }=\max \sum_{v}\left(\Delta \Pi_{j v}(z)+\Delta \pi_{v}(z)\right)=\sum_{v}\left(G_{v}^{\max }-G_{v}^{\min }\right)
$$

A heuristic can be developed to determine the optimal return rate $\tau^{*}$ (egalitarian bargaining solution) by measuring maximum distance between $\Pi$ and $\pi$ for all suppliers. Such points of tangency occur when the concave curve $\Delta \Pi_{v}(\mathrm{x})$ and the convex curve $\Delta \pi_{v}(x)$ with the same slope, where Eqs. (16) and (18) are equal. It yields the optimal value of $\tau$ :

$$
\tau^{3 / 2}=\left[\left((\Delta-A) d+\left((\Delta-A)^{2} d^{2}+8 c^{L} B\right)\right)^{1 / 2}\right] / 4 c^{L}
$$

where $\Delta=\sum_{k} \sum_{l} \Delta_{k l}$ and $c^{L}=\sum_{k} \sum_{l} c_{k l}^{L}$.

To improve the efficiency of the NSGA-CO, filter procedures of a non-egalitarian bargaining solution are introduced in the algorithm to filter out unfeasible combinations of products and suppliers.

Proposition 2. Suppliers are more sensitive to the quantity of demand than manufacturer with consideration of the optimal contract.

Based on the Eqs. (14) and (15), the first-order derivative of demand of the function of suppliers and manufacturer are obtained and denoted as $\Delta$ and $A$, respectively. As discussed before, the parameter $\Delta$ is considerably larger than the parameter $A$, which means the suppliers are more susceptible to the fluctuating demand. This is why the manufacturer always plays the role of leader and suppliers act as followers in the Stackelberg game for PFD as mentioned in Section 2. The manufacturer can influence the suppliers' choice of their remanufacturing effort level by determining the platform components' outsourcing. This proposition will be further discussed in the Section 5. In the next section, another case scenario is used to illustrate how the heuristics are used to determine the optimal return rate $\tau^{*}$ with considering all suppliers.

\subsubsection{Case scenario 2}

Considering one more supplier (B) in the case 1, supplier (B) sets parameter $c^{L}$ as $\$ 760$ and $\Delta$ as \$29. Based on Eqs. (20) and (21), we can find out the feasible set of discounts as shown in Fig. 5. For instance, when the manufacturer decides on the return rate of the used product variant $\tau^{\prime}$ as $0.55, G_{v}^{\max }, G_{A}^{\min }$ and $G_{B}^{\min }$ are set as 873,704 and 771 , respectively. By using the heuristic approach, the egalitarian bargaining solution can be directly realized by 
measuring maximum range between $\Pi$ and $\pi$ considering the two suppliers. The optimal Payoff (\$289) can be calculated where $\tau^{*}$ is $0.29 . G_{v}^{\max }, G_{A}^{\min }$ and $G_{B}^{\min }$ are set as 1164,1019 and 1020, respectively.

Theoretical analysis shows that the heuristic approach can be easily implemented and is effective to solve the problem. The primary goal of the heuristics is to understand the implications of relationship between manufacturer's return rate and suppliers' discount schedule of remanufacturing.

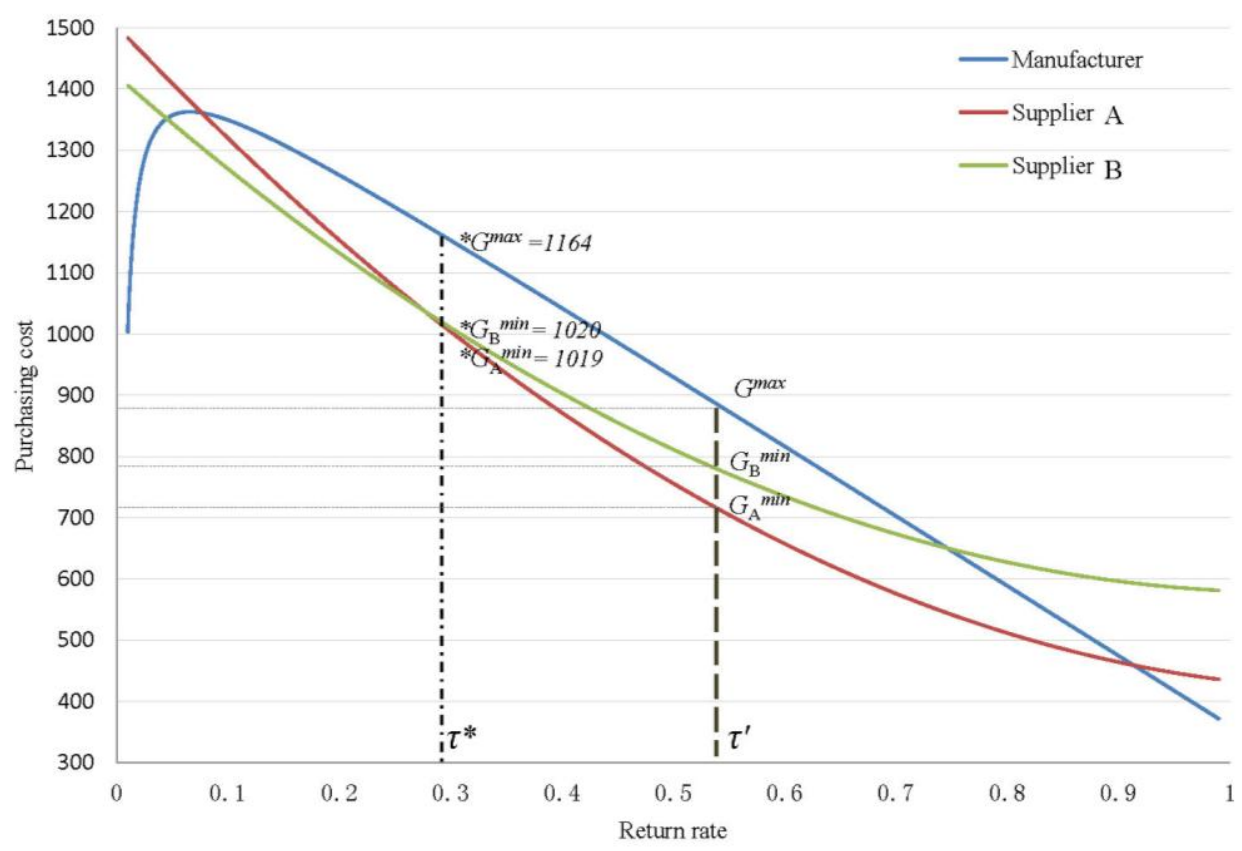

Fig. 5. An example of one manufacturer and two suppliers.

\section{Validation of NSGA-CO}

To validate the effectiveness of the proposed NSGA-CO for solving multi-objective optimization problems, simulation tests were conducted and the simulation results are compared with those generated by NSGA-II. Four different scales of optimization problems, $5 \times 5 \times 5,10 \times 5 \times 5,25 \times 10 \times 10$, and $25 \times 25 \times 25($ RCSs $\times$ alternative components $\times$ suppliers), ranging from medium to large scales, were considered in this study, which are denoted as P1, P-2, P-3, and P-4, respectively. Component price, return rate, payment to the consumer who returns a used product, penalty factor, demand of the product variant, scaling parameter $c^{L}$, unit $\operatorname{cost} c^{m}$ of manufacturing a new product, and the unit cost of remanufacturing $\left(c^{r}\right)$ were generated randomly in the ranges of $[1000,5000],[0.1,0.9],[25,75],[50,500],[25$, 100], [500, 1000], [250, 300], and [150, 250], respectively. For the test problems, the population size was set as 100 , and the iteration was set as 200 . For statistical comparison, all 
tests were executed for 10 times, and solutions are considered as the mean values of the ten runs.

Efficiency of Pareto optimization can also be assessed using the number of evaluations. It is desirable to determine a Pareto front using a small number of evaluations because each evaluation would require a significant amount of CPU time. As mentioned before, the search path taken by the meta-heuristics is generally different in each evaluation and it is quite dependent on its starting conditions. It is necessary to perform multiple runs of the algorithm for each problem in order to obtain the means and variations when comparing the performance between NSGA-CO and NSGA-II. Superimposed results of four evaluations for NSGA-CO and NSGA-II with scale P-4 are presented in Figures 6 (A) to (D) respectively for $1,000,10,000,100,000$, and 1,000,000 evaluations with the population size 100 . When the number of evaluations is less than 1,000 (Fig. 6 A), neither of the algorithms are on the front, though NSGA-CO is closer to the front than NSGA-II. After 10,000 evaluations (Fig. 6 B), NSGA-CO is nearly converged to the Pareto front, while NSGA-II remains relatively far from the front. After 100,000 evaluations (Fig. 6 C), NSGA-CO is near the true front, but has not yet reached it, while the front of NSGA-II is relatively far from the true front. After 1,000,000 evaluations (Fig. 6 D), NSGA-CO is essentially on or very close to the true front for all of the runs, and NSGA-II is gradually approaching to the front. In all evaluations, it can be found that NSGA-CO significantly outperformed the NSGA-II algorithms. In addition, it can be noted that more solutions were generated based on NSGA-CO compared with those based on NSGA-II. It is because the proposed NSGA-CO is an enhanced version of NSGA-II in which a dynamic cooperative game is embedded. In NSGA-CO, the stochastic search function of NSGA-II is still fully used to perform a search for the settings of binary variables in the first layer. Referring to Eq. (25), if it is satisfied, the feasible set of discounts can be found. Then, in each evaluation, the cooperative negotiation heuristics are applied to ensure the improvement of the joint payoff of a manufacturer and suppliers and determine more settings of continuous decision variables compared with the NSGA-II's stochastic search for approximate integer solutions. 


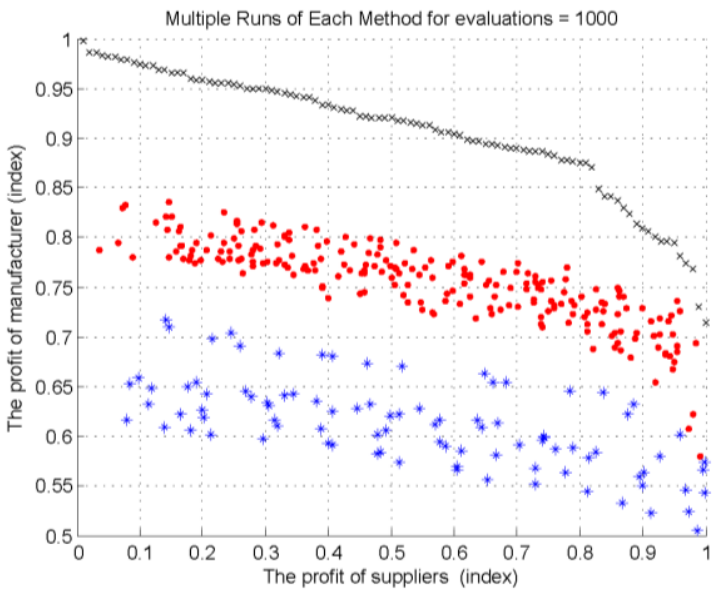

(A)

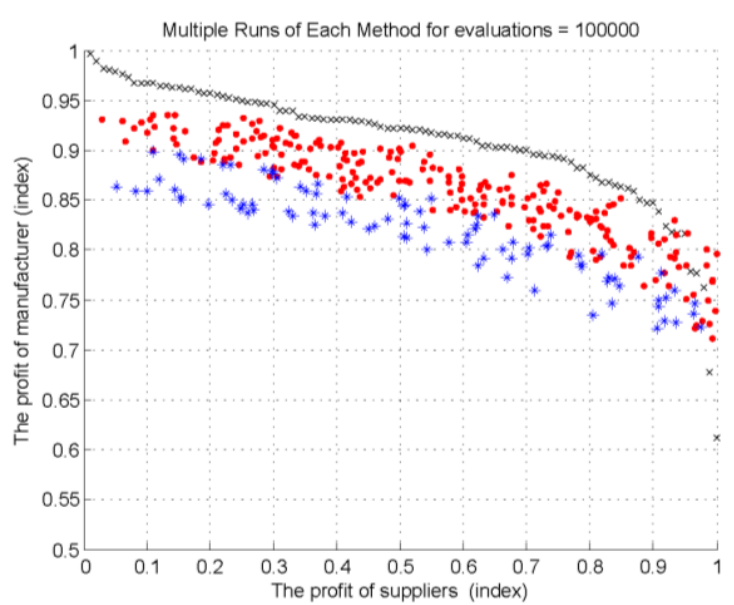

(D)

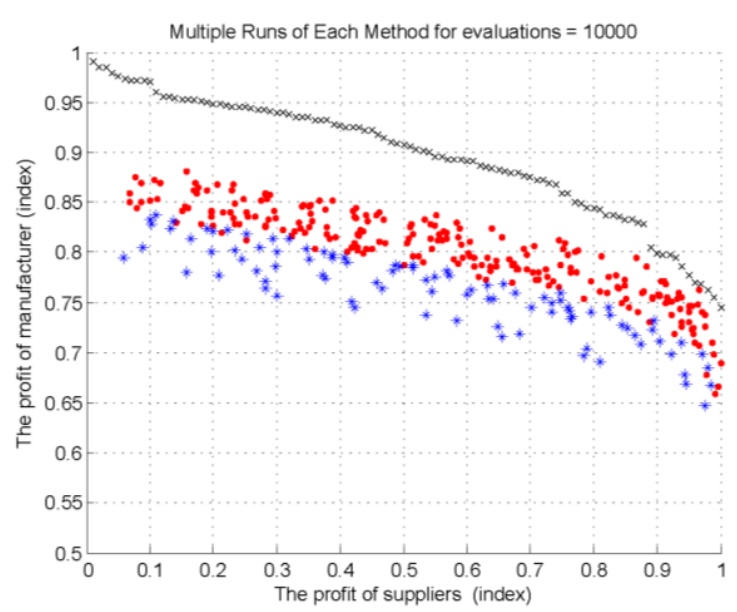

(B)

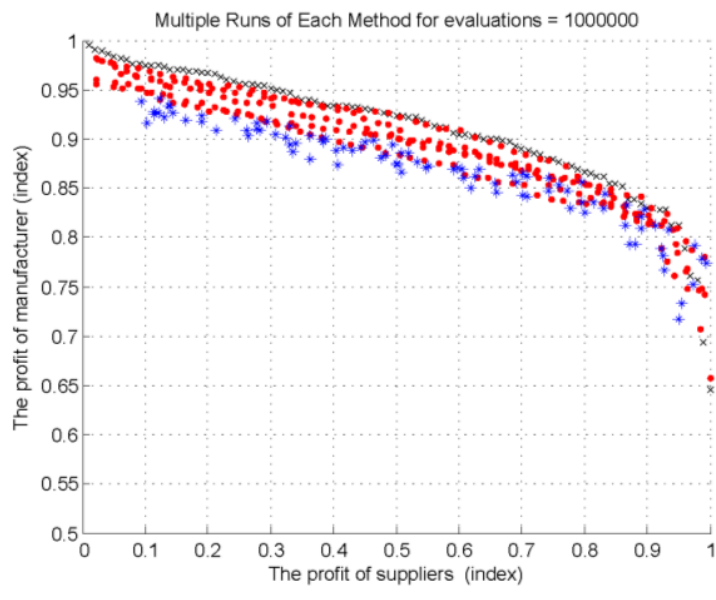

(C)

$\times$ True Front $\quad \cdot$ NSGA-CO $\quad *$ NSGA-II

Fig. 6. Superimposed results with increasing evaluations.

To conduct more rigorous benchmark between NSGA-CO and NSGA-II in solving multiobjective optimization problems, the following representative parameter design were adopted to perform various simulation tests: 1 ) The crossover rates $0.3,0.5$, and 0.7 are used, while keeping the mutation rate as $0.04 ; 2$ ) The mutation rates $0.01,0.04$, and 0.08 are used, while keeping the crossover rate as 0.7 . Since the true Pareto-optimal solution set termed as $P_{t}$ is not known and never presented for multi-objective optimization problems, the best solution set of all tests in this research with adopting iteration times of 10000 generations is treated as $P_{t}$.

There are two goals in multiobjective optimization which are convergence to a Pareto optimal set and maintenance of diversity in solutions of the Pareto optimal set. Many 
performance metrics have been suggested for multiobjective optimization such as epsilon indicator, convergence metric, diversity metric and R-metric [39, 42]. Two metrics, convergence metric $\gamma$ and diversity metric $\psi$ [30], were adopted in this study to compare the performance of the two algorithms in solving multi-objective optimization problems. It is because they can provide direct measures on how good a multi-objective evolutionary algorithm achieves the two goals and were commonly used in previous studies to compare the performance of multi-objective evolutionary algorithms. Convergence metric $\gamma$ measures the extent of convergence to the $P F_{t}$ which is defined as:

$$
\gamma=\frac{1}{N} \sum_{n=1}^{N} \min \left(d_{n m}, \forall m=1, \ldots, M\right)
$$

where $P F_{t}$ is true Pareto front; $P F_{n}$ is the Pareto front of the $n$-th test; $N$ and $M$ represent the number of vectors belongs to $P F_{n}$ and $P F_{t}$, respectively. The expression $\min \left(d_{n m}, \forall m=1, \ldots, M\right)$ represents the minimum Euclidean Distance of vector $n$ belongs to $P F_{n}$ from all vectors belongs to $P F_{t}$. The smaller the value of the metric indicates the better the convergence toward $P F_{t}$.

Diversity metric $\psi$ is a value measuring the spread (distribution) of vectors throughout $P F_{n}$. Because the boundary of $P F_{n}^{\prime}$ 's "beginning" and "end" are known, Diversity metric $\psi$ can be employed to judge how well $P F_{n}$ is distributed which is defined as:

$$
\psi=\left(d_{f}+d_{l}+\sum_{\bar{i}=1}^{N-1}\left|d_{i}-\bar{d}\right|\right) /\left(d_{f}+d_{l}+(N-1) \bar{d}\right.
$$

where Euclidean distance $d_{i}$ between consecutive vectors belongs to $P F_{n}$, and $\bar{d}$ represents the average distance of $d_{i}$; Parameters $d_{f}$ and $d_{l}$ respectively are the Euclidean distances between the extreme vectors of $P F_{t}$ and the boundary vectors of $P F_{n}$.

Simulation tests based on NSGA-II and NSGA-CO were conducted and their statistical results of convergence and diversity metrics are shown in Tables 1 to 4 .

\begin{tabular}{|c|c|c|c|c|c|c|c|c|}
\hline \multirow{2}{*}{\multicolumn{2}{|c|}{ Parameter }} & \multicolumn{7}{|c|}{ Scale of optimization problems (modules $\times$ alternative components $\times$ suppliers) } \\
\hline & & P-1 $(5 \times 5 \times 5)$ & \multicolumn{2}{|c|}{$\mathrm{P}-2(10 \times 5 \times 5)$} & \multicolumn{2}{|c|}{ P-3 $(25 \times 10 \times 10)$} & \multicolumn{2}{|c|}{ P-4 $(25 \times 25 \times 25)$} \\
\hline & & Mean SD & Mean & SD & Mean & SD & Mean & SD \\
\hline \multirow{3}{*}{ Mutation } & 0.01 & 13.84 & 15.91 & 4.67 & 27.82 & 6.91 & 38.61 & 8.62 \\
\hline & 0.04 & 12.55 & 15.97 & 7.67 & 25.41 & 7.97 & 31.78 & 10.23 \\
\hline & 0.08 & 14.31 & 17.12 & 7.96 & 28.25 & 8.31 & 40.82 & 11.96 \\
\hline Crossover & 0.3 & 14.18 & 32.43 & 11.82 & 49.51 & 16.35 & 75.35 & 38.14 \\
\hline
\end{tabular}

Table 1. Statistical results of convergence metrics for NSGA-II 


\begin{tabular}{lllllllll}
0.5 & 13.84 & 6.85 & 26.75 & 10.57 & 42.87 & 17.38 & 68.82 & 31.52 \\
\hline 0.7 & 13.52 & 5.68 & 26.84 & 11.85 & 40.56 & 15.82 & 55.41 & 25.15
\end{tabular}

where mean and SD represent the mean values and standard deviation of the convergence metrics, respectively.

Table 2. Statistical results of convergence metric for NSGA-CO

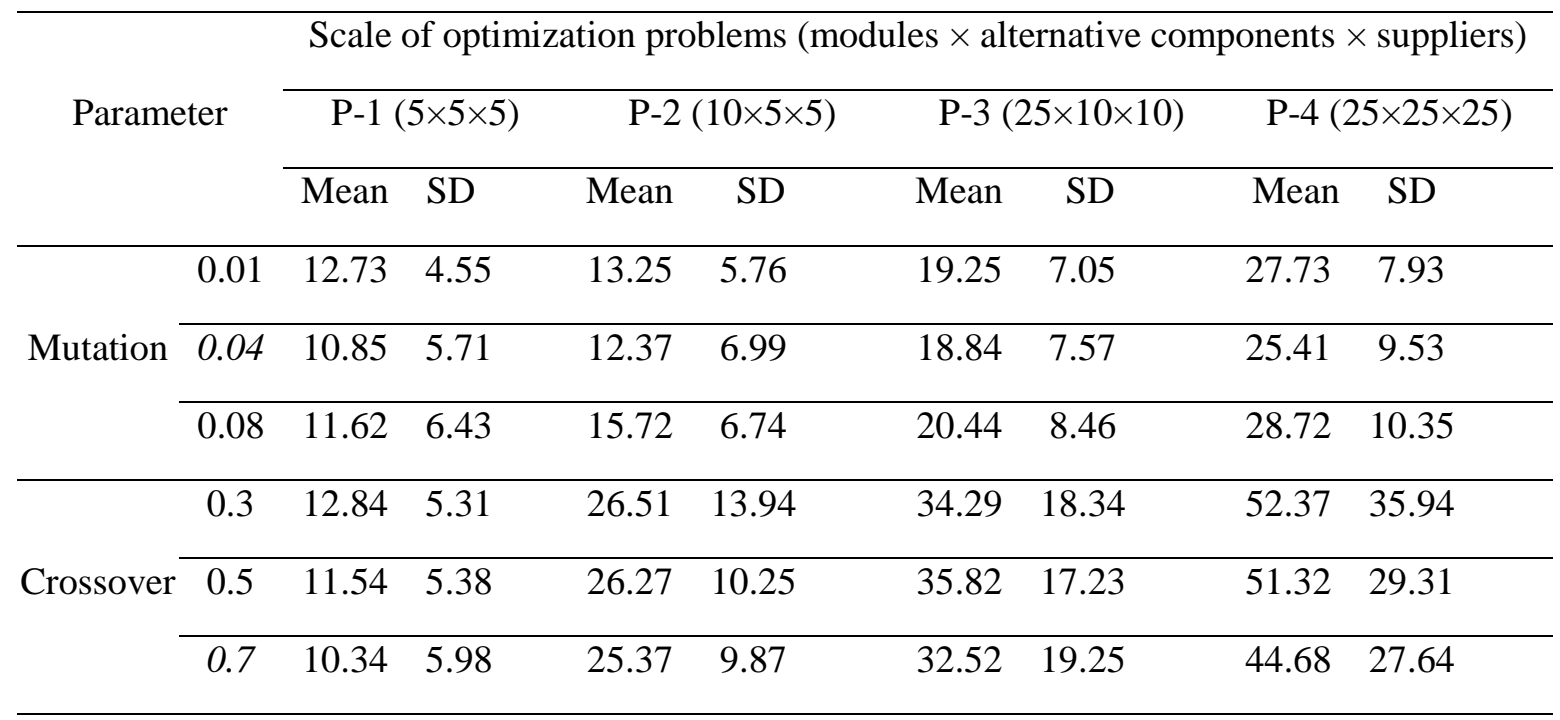

Table 3. Statistical results of diversity metric for NSGA-II

\begin{tabular}{|c|c|c|c|c|c|c|c|}
\hline \multirow{2}{*}{\multicolumn{2}{|c|}{ Parameter }} & \multicolumn{6}{|c|}{ Scale of optimization problems (modules $\times$ alternative components $\times$ suppliers) } \\
\hline & & $\mathrm{P}-1(5 \times 5 \times 5)$ & \multicolumn{2}{|c|}{$\mathrm{P}-2(10 \times 5 \times 5)$} & \multicolumn{2}{|c|}{ P-3 $(25 \times 10 \times 10)$} & P-4 $(25 \times 25 \times 25)$ \\
\hline & & Mean SD & Mean & SD & Mean & SD & Mean SD \\
\hline \multirow{3}{*}{ Mutation } & 0.01 & $0.43 \quad 0.11$ & 0.72 & 0.15 & 0.85 & 0.32 & 0.55 \\
\hline & 0.04 & $0.41 \quad 0.26$ & 0.71 & 0.12 & 0.83 & 0.29 & 0.84 \\
\hline & 0.08 & $0.36 \quad 0.16$ & 0.59 & 0.42 & 0.77 & 0.61 & 0.85 \\
\hline \multirow{3}{*}{ Crossover } & 0.3 & $\begin{array}{ll}0.45 & 0.07\end{array}$ & 0.85 & 0.12 & 0.69 & 0.09 & 0.87 \\
\hline & 0.5 & $\begin{array}{ll}0.47 & 0.23\end{array}$ & 0.79 & 0.28 & 0.72 & 0.75 & 0.35 \\
\hline & 0.7 & $\begin{array}{ll}0.45 & 0.54\end{array}$ & 0.78 & 0.55 & 0.68 & 0.68 & 0.48 \\
\hline
\end{tabular}

where mean and SD represent mean and standard deviation of the diversity metric. 
Table 4. Statistical results of diversity metric for NSGA-CO

\begin{tabular}{cccccccccc}
\hline & \multicolumn{3}{c}{ Scale of optimization problems (modules $\times$ alternative components $\times$ suppliers) } \\
\cline { 2 - 10 } Parameter & \multicolumn{2}{c}{ P-1 $(5 \times 5 \times 5)$} & \multicolumn{2}{c}{ P-2 $(10 \times 5 \times 5)$} & P-3 $(25 \times 10 \times 10)$ & P-4 $(25 \times 25 \times 25)$ \\
\cline { 2 - 10 } & & Mean & SD & Mean & SD & Mean & SD & Mean & SD \\
\hline \multirow{3}{*}{ Mutation } & 0.01 & 0.39 & 0.19 & 0.61 & 0.2 & 0.81 & 0.41 & 0.91 & 0.35 \\
\cline { 2 - 10 } & 0.04 & 0.37 & 0.11 & 0.62 & 0.17 & 0.75 & 0.37 & 0.85 & 0.53 \\
\cline { 2 - 10 } & 0.08 & 0.41 & 0.23 & 0.77 & 0.52 & 0.89 & 0.76 & 0.96 & 0.75 \\
\hline \multirow{3}{*}{ Crossover } & 0.3 & 0.41 & 0.35 & 0.79 & 0.26 & 0.88 & 0.21 & 0.91 & 0.38 \\
\cline { 2 - 10 } & 0.5 & 0.49 & 0.14 & 0.81 & 0.74 & 0.78 & 0.55 & 0.81 & 0.41 \\
\hline & 0.7 & 0.32 & 0.21 & 0.64 & 0.31 & 0.71 & 0.38 & 0.82 & 0.56 \\
\hline
\end{tabular}

From Tables 1 and 2, it can be noted that the mean values of convergence metric of NSGA$\mathrm{CO}$ are smaller than those of NSGA-II for all the simulation tests. Regarding SD, difference of SD values between NSGA-II and NSGA-CO overall is small and no consistent pattern can be seen. However, it can be seen that for large scale problem (i.e. P-4), nearly all the SD values of NSGA-CO are smaller than those of NSGA-II. The simulation results indicated that NSGA-CO outperforms NSGA-II in terms of convergence. From Tables 3 and 4, nearly $60 \%$ of the mean values of diversity metric of NSGA-CO is smaller than those of NSGA-II. It can also be noted that NSGA-CO provides relatively better solutions than NSGA-II in mediumscale problems (i.e., P-1 and P-2) in terms of diversity. Thus, it can be said that the performance of NSGA-II and NSGA-CO are quite similar in terms of diversity.

\section{Computational experiments and effect analysis}

A case study on product family design of printing calculators conducted in our previous study [18] was extended in this study by incorporating the supply chain design and remanufacturing issues into product family design. The extended case study was then adopted to evaluate the effectiveness of NSGA-CO in solving the integrated product family and supply chain design problem and investigate the effects of environmental penalty, quantity of demand and marginal cost of remanufacturing on used product return rate, manufacturers' and suppliers' profits and joint payoff. Some results of our previous case study, including market segmentation using a C-means clustering method and the estimation of part-worth utilities 
using least-square linear regression, were adopted in the extended case study. Suppose an electronic product manufacturer is considering to develop a family of new printing calculators that would involve both new and remanufactured products. The printing calculators were designed in a modular structure and the modules are provided by suppliers. Six RCSs were defined which are product case (RCS1), display system (RCS2), keyboard (RCS3), function switch (RCS4), printing system (RCS5), and electronic system (RCS6). The display system (RCS2) and printing system (RCS5) were identified to be the ones where remanufactured modules can be provided. A two-echelon supply chain model as shown in Fig. 2 is considered in the case.

A conjoint survey based on an L25 orthogonal array was conducted. Based on the survey results, three segments were identified using a C-means clustering method and the part-worth utility of each RCS component of individual segments was estimated using a least-square linear regression method. The third to fifth columns of Table 5 show the results of the regression.

Table 5. Parameters of each component for each RCS

\begin{tabular}{|c|c|c|c|c|c|c|}
\hline RCS & $\begin{array}{c}\text { Component } \\
\text { (price, \$) }\end{array}$ & $\begin{array}{c}\text { Part worth } \\
\text { utility of } \\
\text { Segment } 1(\$)\end{array}$ & $\begin{array}{c}\text { Part worth } \\
\text { utility of } \\
\text { Segment } 2(\$)\end{array}$ & $\begin{array}{c}\text { Part worth } \\
\text { utility of } \\
\text { Segment } 3(\$)\end{array}$ & $\begin{array}{c}\text { Variable } \\
\text { unit cost }(\$)\end{array}$ & $\begin{array}{c}\text { Remanufac } \\
\text { turing unit } \\
\text { cost }(\$)\end{array}$ \\
\hline \multirow{4}{*}{1} & C11 (3.2) & 6.1 & 4.7 & 4.5 & 0.3 & - \\
\hline & C12 (3.5) & 6.3 & 4.9 & 4.5 & 0.3 & 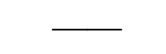 \\
\hline & C13 (4.1) & 6.5 & 5.3 & 5.3 & 0.5 & 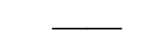 \\
\hline & C14 (4.1) & 6.5 & 6.0 & 4.2 & 0.5 & - \\
\hline \multirow{4}{*}{2} & $C 21(6.5)$ & 16.5 & 16.9 & 16.2 & 0.2 & 2.5 \\
\hline & C22(7.2) & 16.6 & 18.2 & 16.2 & 0.3 & 2.8 \\
\hline & $C 23(6.7)$ & 16.6 & 16.8 & 16.6 & 0.3 & 2.8 \\
\hline & C24(6.8) & 16.6 & 16.8 & 18.0 & 0.4 & - \\
\hline \multirow{3}{*}{3} & C31(1.7) & 2.6 & 4.3 & 3.2 & 0.2 & $\bar{\square}$ \\
\hline & C32(1.9) & 2.7 & 4.5 & 3.6 & 0.2 & - \\
\hline & C33(1.6) & 2.6 & 4.1 & 4.3 & 0.3 & - \\
\hline \multirow{3}{*}{4} & C41(0.3) & 0.6 & 0.6 & 0.4 & 0.1 & $\bar{\square}$ \\
\hline & C42(0.4) & 0.6 & 0.8 & 0.4 & 0.1 & $\longrightarrow$ \\
\hline & C43(0.6) & 0.8 & 0.8 & 0.8 & 0.3 & $\longrightarrow$ \\
\hline 5 & C51(6.3) & 12.2 & 11.7 & 8.8 & 0.2 & 1.3 \\
\hline
\end{tabular}




\begin{tabular}{ccccccc}
\hline & C52(6.5) & 12.3 & 12.1 & 10.2 & 0.3 & 1.5 \\
& C53(6.9) & 12.4 & 11.8 & 10.7 & 0.3 & 1.5 \\
\hline \multirow{3}{*}{6} & C61(4.7) & 8.5 & 6.5 & 6.5 & 0.1 & - \\
& C62(5.0) & 8.6 & 6.5 & 6.5 & 0.1 & - \\
\hline
\end{tabular}

In this case, the components C21 to C23 of the second RCS and components C51 to C53 of the fifth RCS were identified to be remanufacturable and the estimated remanufacturing unit costs are shown in the sixth column of Table 5.

To analyze the effects of environmental penalty, quantity of demand and the marginal cost of remanufacturing on the used product return rate, manufacturers' and suppliers' profits and joint payoff, an optimization model based on Eqs. (1) to (6) was formulated and solved by NSGA-CO. A series of computational experiments were conducted based on the formulated model to analyze the effects of the marginal cost of remanufacturing, quantity of demand and environmental penalty. For each experiment, NSGA-CO was adopted to solve the problems with the population size, 100, and iteration, 200. Crossover rate and mutation rate were set as 0.7 and 0.04 , respectively, which are the best value of those parameters according to the above studies. The analysis results of the effects of marginal cost of remanufacturing, quantity of demand and environmental penalty factor are described in the following sub-sections.

\subsection{Analysis of the effect of marginal cost of remanufacturing}

Referring to Section 3, the marginal cost of remanufacturing is defined as $\Delta=\left(c^{m}-c^{r}\right)$, where $c^{m}$ denotes the unit cost of manufacturing a new product, and $c^{r}$ is the unit cost of remanufacturing a returned product. Thus, if a supplier invests $c^{L} \tau^{2}$, the unit marginal cost can be reduced by $\Delta$. To analyze the effect of the marginal cost of remanufacturing, the lower and higher boundary of marginal costs were set as $\$ 10$ and $\$ 80$, respectively. The feasible set of discounts for corresponding marginal cost were set as 10, 40 and 80, as shown in Fig. 7 (A), (B) and (C) respectively. With the increasing factor $\Delta$, the coordinated discount prices $G_{v}^{x}$ offered by the supplier decrease and the egalitarian bargaining solution moves down consequentially. It is because the growth of supplier's profit accompanies with the increase of marginal cost of remanufacturing. The egalitarian bargaining solution suggests that the 
players could maximize their utilities subject to the restriction in which they all gain "equally" in the given situation.

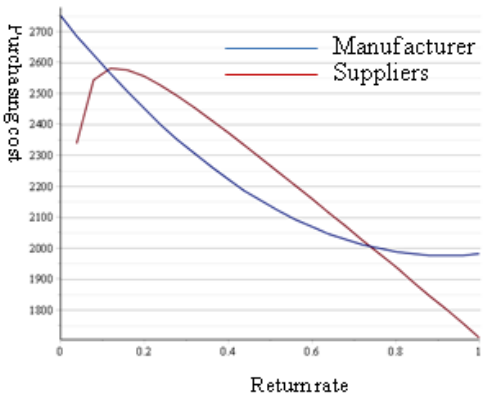

(A)

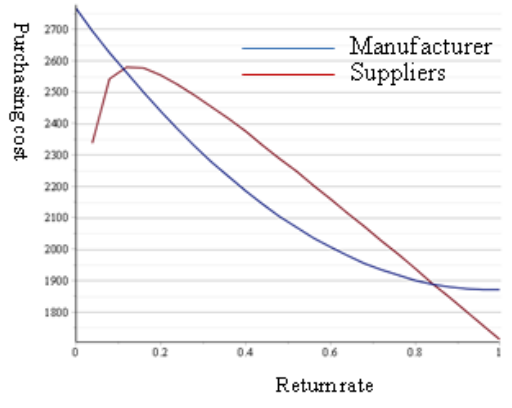

(B)

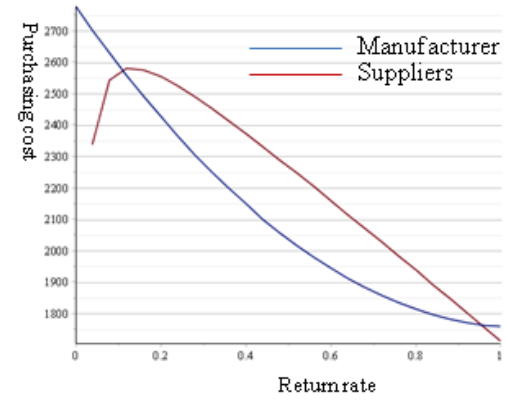

(C)

Fig. 7. The feasible set of different marginal cost.

The mean value of replications is plotted as the final result (See Fig. 8). The optimal manufacturer's profit and the optimal suppliers' profit are derived from the Pareto front to analyze the effects of the return rate and the marginal cost, ceteris paribus, on the remanufacturing.

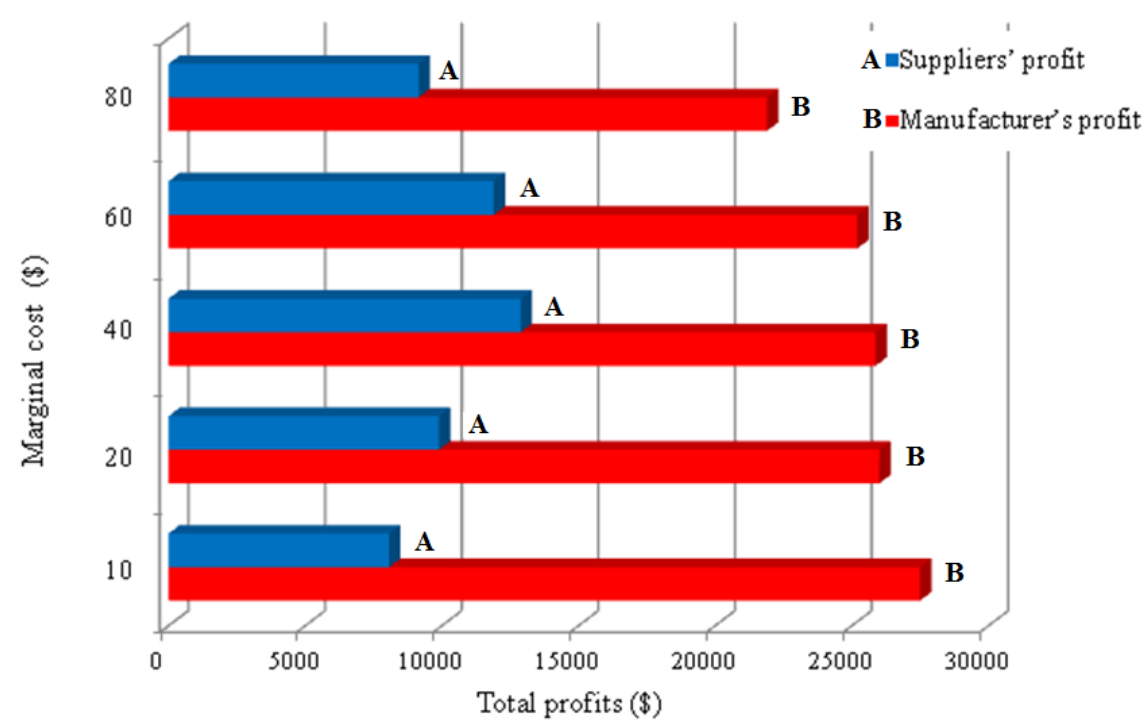

Fig. 8. The impact of the marginal cost.

Two conclusions can be drawn from the results shown in Fig. 7. With the increase of the marginal cost, the optimal return rate increases and manufacturer's profit decreases. The profit of suppliers may increase at the beginning because more incentives are provided to increase return rate; thus production cost decreases. However, the suppliers' profit may not 
continue to increase when marginal cost reaches around $\$ 40$. It is because the cost structure of the investment in the collection of used product is formulated as $c^{L} \tau^{2}$. The diminishing return to investment indicates that a higher collecting cost should be paid to improve the return rate when the return rate reaches the optimal level. Therefore, it can be concluded that the marginal cost saving is a driving force of remanufacturing. However, due to the diminishing return to investment, the optimal return rate always performs a balance between development cost saving and collection investment.

\subsection{Analysis of the effect of demand quantity}

To analyze the effect of the fluctuating demand, four demand levels, 10, 25, 75 and 100 (Unit), are considered. The feasible set of discounts for each demand can be found in Fig. 9 (A), (B), (C) and (D) respectively. With the increasing demand, the average coordinated discount prices offered by the supplier and the manufacturer decrease simultaneously. The optimal return rate increases dramatically from about 0.37 to 0.52 while the joint payoff increases.

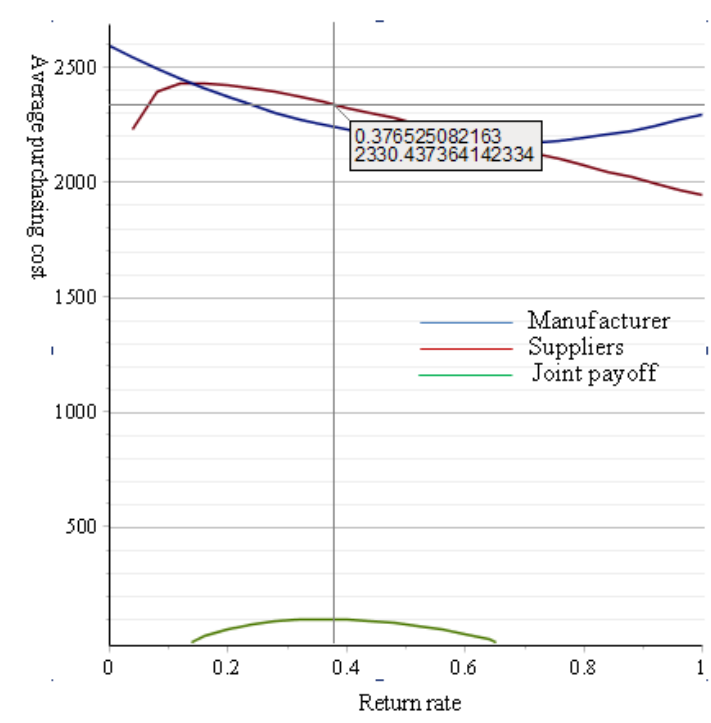

(A)

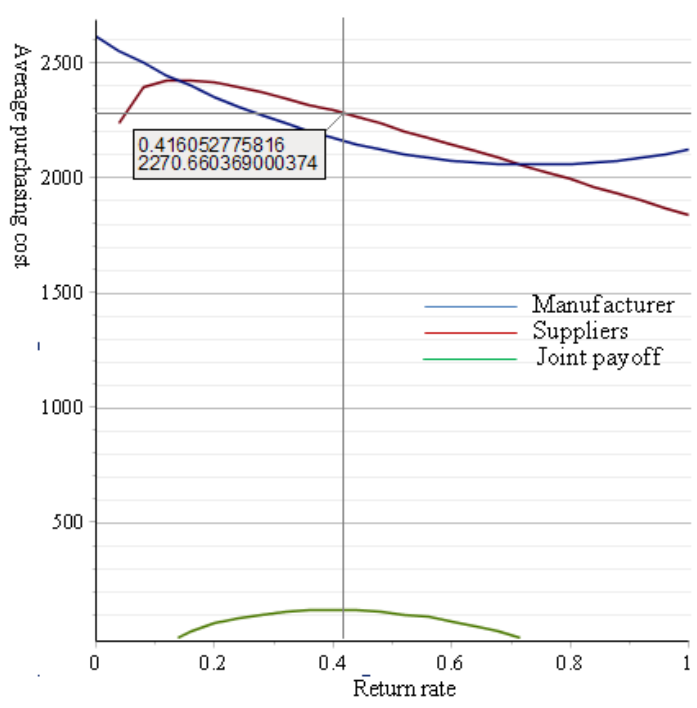

(B) 


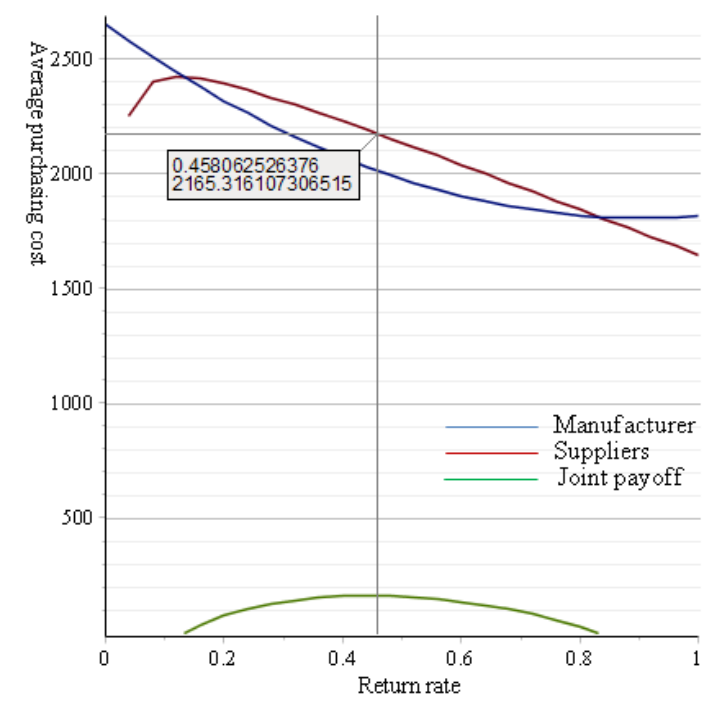

(C)

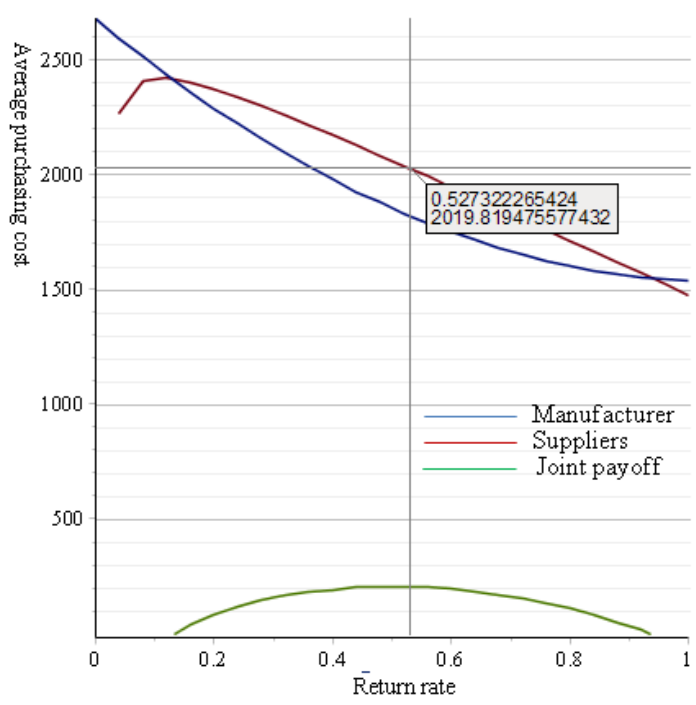

(D)

Fig. 9. The feasible set of different demand levels.

\subsection{Analysis of the effect of environmental penalty factor}

In this section, we further investigated the effect of the environmental penalty on the remanufacturing. As mentioned before, a government policy-maker attempts to increase remanufacturing activity. In this paper, we investigate the effect of implementing penalty system in industries by incorporating a penalty factor $(B)$ into environmental (green) supply chain performance metric.

The feasible set of discounts, where the environmental penalty factor $(B)$ is set as 300 and 100, can be found in Fig. 10 (A) and (B) respectively. With the decreasing value of factor $B$, the coordinated discount prices $\left(G_{v}^{x}\right)$ offered by the manufacturer tamp down and the egalitarian bargaining solutions move down consequentially. Obviously, it is due to the fact that the manufacturer has less pressure on the remanufacturing and thus tries to reduce the purchase cost.
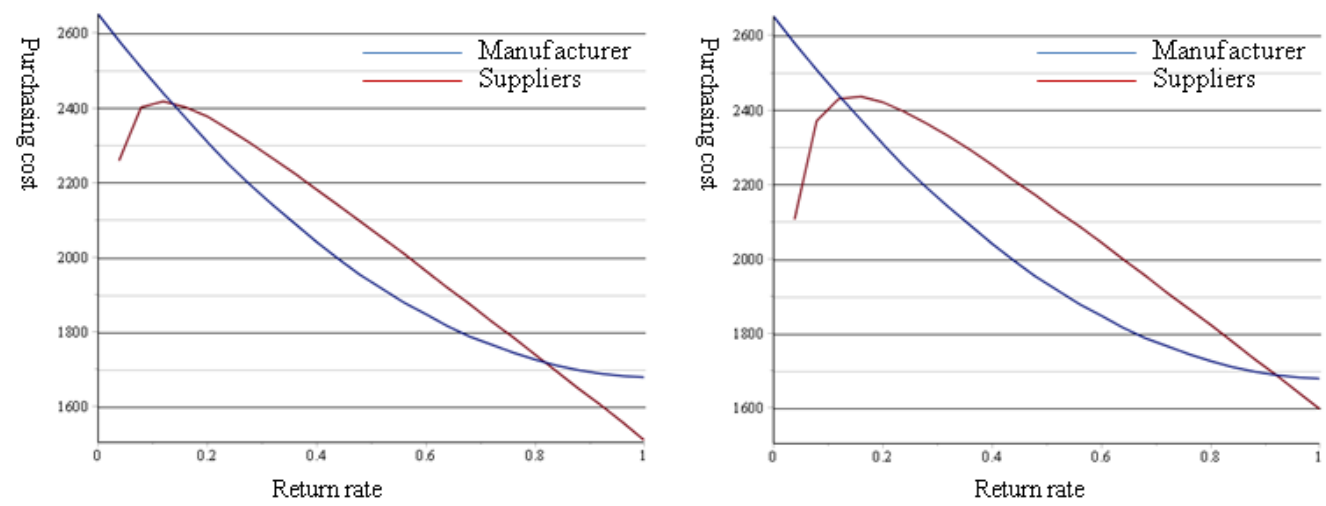
Fig. 10. The feasible set of different environmental penalty.

Fig. 11 (A) and (B) show the nondominated solutions with the environmental penalty factors 300 and 100, respectively. The figures show that a larger environmental penalty would lead to less total profit to be obtained. Referring to Eq. (25), $B$ should be set as not less than $(\Delta-A)^{2} d^{2} / 8 c^{L}$. Otherwise, no feasible set of discounts $\left(X_{z o n}\right)$ would be found.

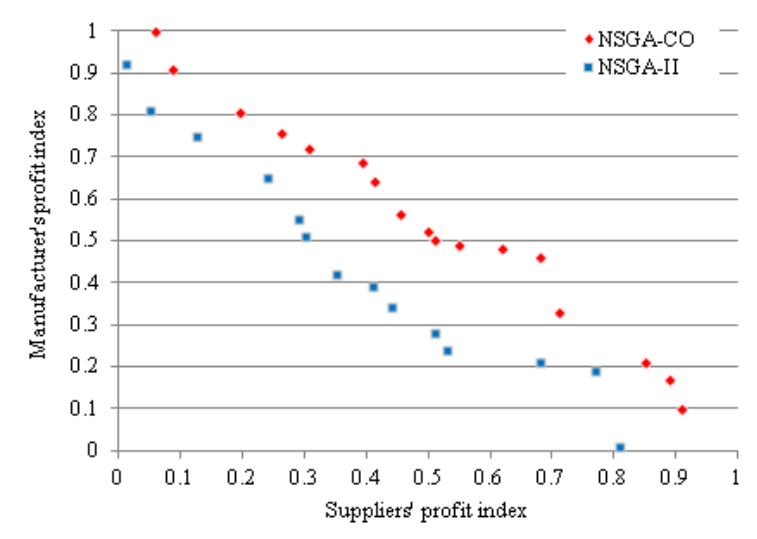

(A)

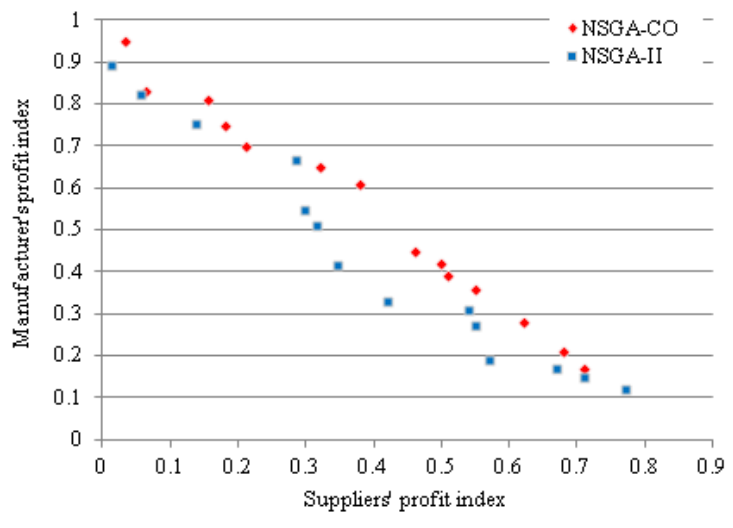

(B)

Fig. 11. The impact of the of the environmental penalty.

The analyses results indicate that the environmental penalty factor plays an important role to remanufacturing implementation. The environmental penalty factor is proportional to the demand quantity of components; and is inversely proportional to the amount of investment in extra return rates. Regarding the demand quantity, suppliers are more sensitive than the manufacturer. Furthermore, the marginal cost saving of remanufacturing is a fundamental driving force of the collection of used product. However, due to diminishing return to investment, the optimal return rate should be determined with consideration of a trade-off between development cost saving and collection investment.

\section{Conclusions and Future Research}

In this paper, formulation of a multi-objective optimization model for integrated product family and supply chain design with consideration of remanufacturing was presented. To solve the large-scale optimization model which involves mixed discrete-continuous decision variables, a novel solving algorithm, cooperative negotiation embedded NSGA-II (NSGA- 
$\mathrm{CO}$ ), is proposed. To validate the effectiveness of the proposed NSGA-CO in solving multiobjective optimization problems, simulation tests on various sizes of the problem were conducted. The test results indicated that NSGA-CO outperforms NSGA-II in terms of convergence while they perform similarly in terms of diversity. With the formulated optimization problem and NSGA-CO, a case study of integrated product family and supply chain design for printing calculators was conducted to investigate the effects of environmental penalty, quantity of demand and marginal cost of remanufacturing on used product return rate, manufacturers' and suppliers' profits and joint payoff.

The manufacturer undertakes the collection of used products in the reverse channel structure. To a larger extent, there are alternative scenarios in the industry that would be studied in future: (1) manufacturer can provide suitable incentives to an existing retailer who already has a distribution channel to induce the collection, or (2) suppliers can subcontract the collection activity to a third party. On the other hand, this study can be extended by incorporating non-cooperative negotiation into NSGA-CO.

\section{Acknowledgement}

The work described in this paper was supported by a grant from The Hong Kong Polytechnic University (Project No. G-YBDZ) and the National Science Foundation of China (NSFC 71301107, 71671028).

\section{References}

[1] Z. Rob, K. Harold, Strategic response to EEE returns: Product eco-design or new recovery processes, Eur. J. Oper. Res. 191 (3) (2008) 1206-1222.

[2] R. Subramanian, M.E. Ferguson, L.B. Toktay, Remanufacturing and the component commonality decision, Prod. Oper. Manag. 22 (1) (2013) 36-53.

[3] W. Ho, X. Xu, P.K. Dey, Multi-criteria decision making approaches for supplier evaluation and selection: A literature review, Eur. J. Oper. Res. 202 (1) (2010) 16-24.

[4] R.R. Hussien, H.M. Abdelsalam, Joint supplier selection and product family optimization in supply chain design: A literature review, Int. J. Comput. Sci. Iss. 12 (2) (2015) 200-206.

[5] S. Gupta, V. Krishnan, Integrated component and supplier selection for a product family, Prod. Oper. Manag. 8 (2) (1999) 163-182.

[6] X.G. Luo, C.K. Kwong, J.F. Tang, S.F. Deng, J. Gong, Integrating supplier selection in optimal product family design, Int. J. Prod. Res. 49 (14) (2011) 4195-4222.

[7] Y. Cao, X.G. Luo, C.K. Kwong, J.F. Tang, W. Zhou, Joint optimization of product family 
design and suppler selection under multinomial logit consumer choice rule, Concurrent Eng-Res. A 20 (4) (2012) 335-347.

[8] N.R. Balakrishnan, A.K. Chakravarty, Product design with multiple suppliers for component variants, Int. J. Prod. Econ. 112 (2) (2008) 723-741.

[9] G.Q. Huang, X.Y. Zhang, V.H.Y. Lo, Integrated configuration of platform products and supply chains for mass customization: A game-theoretic approach, IEEE Trans. Eng. Manage. 54 (1) (2007) 156-171.

[10] X.Y. Zhang, G.Q. Huang, P.K. Humphreys, V. Botta-Genoulaz, Simultaneous configuration of platform products and manufacturing supply chains, Int. J. Prod. Res. 46 (21) (2008) 6137-6162.

[11] S. Deng, R. Aydin, C.K. Kwong, Y. Huang, Integrated product line design and supplier selection: A multi-objective optimization paradigm, Comput. Ind. Eng. 70 (1) (2014) 150158.

[12] D. Yang, J. Jiao, Y. Ji, G. Du, P. Helo, A. Valente, Joint optimization for coordinated configuration of product families and supply chains by a leader-follower Stackelberg game, Eur. J. Oper. Res. 246 (1) (2015) 263-280.

[13] B. Baud-Lavigne, B. Agard, B. Penz, Simultaneous product family and supply chain design: An optimization approach, Int. J. Prod. Econ. 174 (2016) 111-118.

[14] Q.L. Xu, S.K. Ong, A.Y.C.Nee, Evaluation of product performance in product family design re-use, Int. J. Prod. Res. 45 (18-19) (2007) 4119-4141.

[15] G. Ferrer, J.M. Swaminathan, Managing new and differentiated remanufactured products, Eur. J. Oper. Res. 203 (2010) 370-379.

[16] J. Wang, J. Zhao, X. Wang, Optimum policy in hybrid manufacturing/remanufacturing system, Comput. Ind. Eng. 60 (3) (2011) 411-419.

[17] H. Metta, F. Badurdeen, Integrating sustainable product and supply chain design: Modeling issues and challenges, IEEE Trans. Eng. Manage. 60 (2) (2013) 438-446.

[18] Z. Wu, C.K. Kwong, C.K.M. Lee, J. Tang, Joint decision of product configuration and remanufacturing for product family design. Int. J. Prod. Res. 54(15) (2016) 4689-4702.

[19] Y. He, Acquisition pricing and remanufacturing decisions in a closed-loop supply chain, Int. J. Prod. Econ. 163 (2015) 48-60.

[20] J. Tao, S. Yu, Incorporating reuse and remanufacturing in product family planning, in: M. Matsumuto, Y. Umeda, K. Masui, S. Fukushige (Eds.), Design for innovative value towards a sustainable society, Springer, Netherlands, 2012, pp. 795-800.

[21] R. Aydin, C.K. Kwong, P. Ji, Coordination of the closed-loop supply chain for product 
line design with consideration of remanufactured products, J. Clean. Prod. 114 (2015) 286-298.

[22] J. Shi, G. Zhang, J. Sha, Optimal production planning for a multi-product closed loop system with uncertain demand and return, Comput. Oper. Res. 38 (3) (2011) 641-650.

[23] Z.Q. Wu, J.F. Tang, C.K. Kwong, C.Y. Chan, An optimization model for reuse scenario selection considering reliability and cost in software product line development, Int. J. Info. Tech. Dec. Mak. 10 (5) (2011) 811-841.

[24] R.C. Savaskan, S. Bhattacharya, L.N.V. Wassenhove, Closed-loop supply chain models with product remanufacturing, Manage. Sci. 50 (2) (2004) 239-252.

[25] C.H. Wu, OEM product design in a price competition with remanufactured product, Omega-Int. J. Manage. Sci. 41 (2) (2013) 287-298.

[26] C.H. Wu, Product-design and pricing strategies with remanufacturing, Eur. J. Oper. Res. 222 (2) (2012) 204-215.

[27] F.M. Defersha, M. Chen, A linear programming embedded genetic algorithm for an integrated cell formation and lot sizing considering product quality, Eur. J. Oper. Res. 187 (1) (2008) 46-69.

[28] C.K. Kwong, X.G. Luo, J.F. Tang, A multiobjective optimization approach for product line design, IEEE Trans. Eng. Manage. 58 (1) (2011) 97-108.

[29] N. Gatti, F. Amigoni, An approximate Pareto optimal cooperative negotiation model for multiple continuous dependent issues, International Conference on Intelligent Agent Technology, France, 2005, pp. 565-571.

[30] K. Deb, A. Pratap, S. Agarwal, T. Meyarivan, A fast and elitist multiobjective genetic algorithm: NSGA-II, IEEE Trans. Evolut. Comput. 6 (2) (2002) 182-197.

[31] C. Coello, Evolutionary multi-objective optimization: A historical view of the field, IEEE Comput. Intell. M. 1 (1) (2006) 28-36.

[32] X. Wang, J.D. Camm, D.J. Curry, A branch-and-price approach to the share-of-choice product line design problem, Manage. Sci. 55 (10) (2009) 1718-1728.

[33] T.H. Hsu, K.M. Chu, H.C. Chan, The fuzzy clustering on market segment, The Ninth IEEE International Conference on Fuzzy Systems, San Antonio, 2000, pp. 621-626.

[34] G.E. Fruchter, S. Kalish, Closed-Loop Advertising Strategies in a Duopoly, Manage. Sci. 43 (1) (1997) 54-63.

[35] H. Zhao, Raising awareness and signaling quality to uninformed consumers: A priceadvertising model, Market. Sci. 19 (4) (2000) 390-396.

[36] Z.K. Weng, Channel coordination and quantity discounts, Manage. Sci. 41 (9) (1995) 
1509-1522.

[37] B.M. Beamon, Designing the green supply chain, Logist. Inform. Manage. 12 (4) (1999) 332-342.

[38] A.A. Hervani, M.M. Helms, J. Sarkis, Performance measurement for green supply chain management, Benchmark. Int. J. 12 (4) (2005) 330-353.

[39] K. Deb, Multiobjective optimization using evolutionary algorithms, Wiley, Chichester, U.K., 2001.

[40] N. Lalonde, Multiobjective optimization algorithm benchmarking and design under parameter uncertainty, Dissertation, Queen's University, Canada; 2009.

[41] D.E. Goldberg, Genetic algorithms in search, optimization, and machine learning, Addison-Wesley, Boston, 1989.

[42] N. Srinivas, K. Deb, Multiobjective optimization using nondominated sorting in genetic algorithms, Evol. Comput. 2 (3) (1994) 221-248.

[43] E. Kalai, Proportional solutions to bargaining situations: Interpersonal utility comparisons, Econometrica. 45 (7) (1977) 1623-1630. 


\section{Appendix A. A flowchart of NSGA-II}

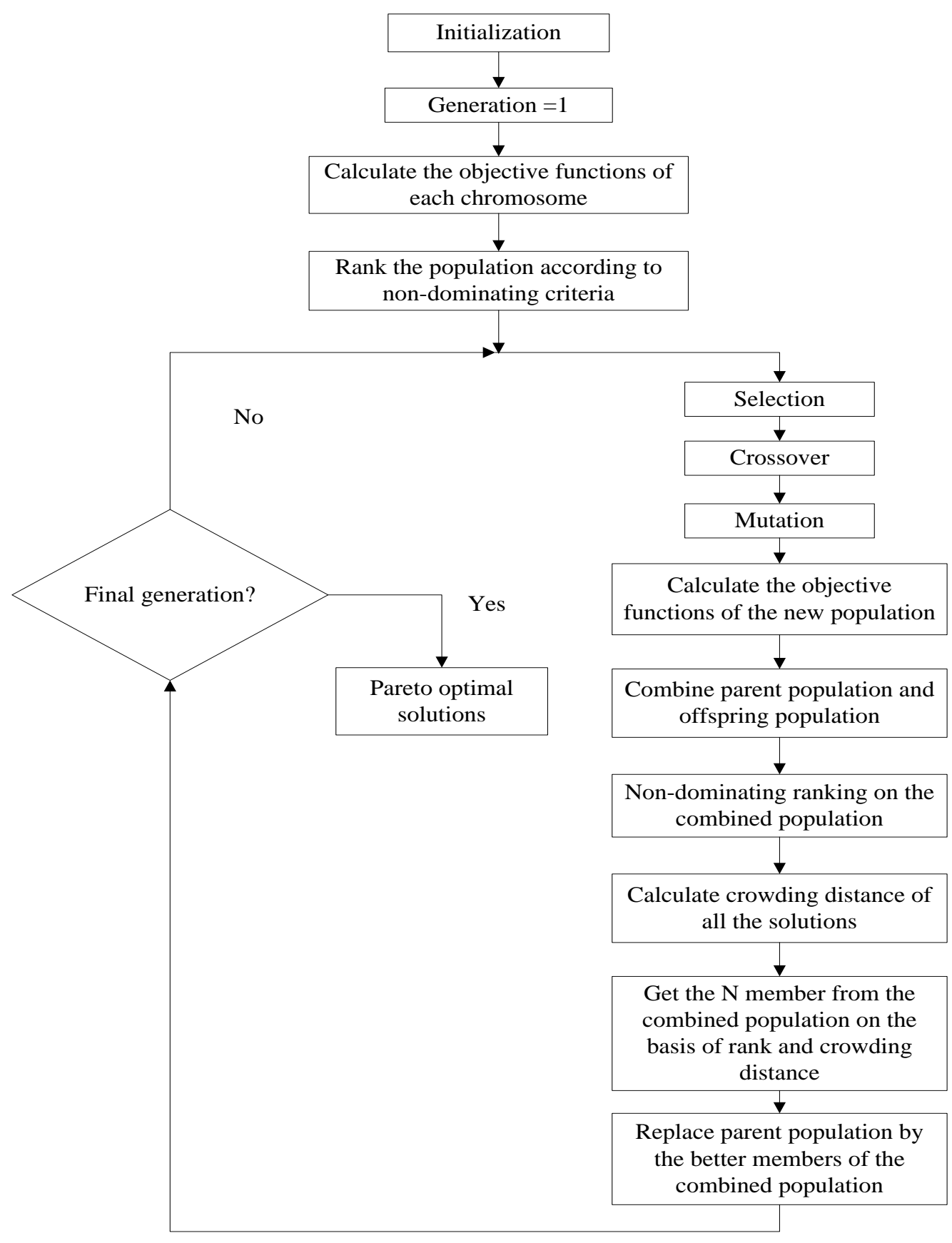

Fig. A1. A flowchart of NSGA-II. 


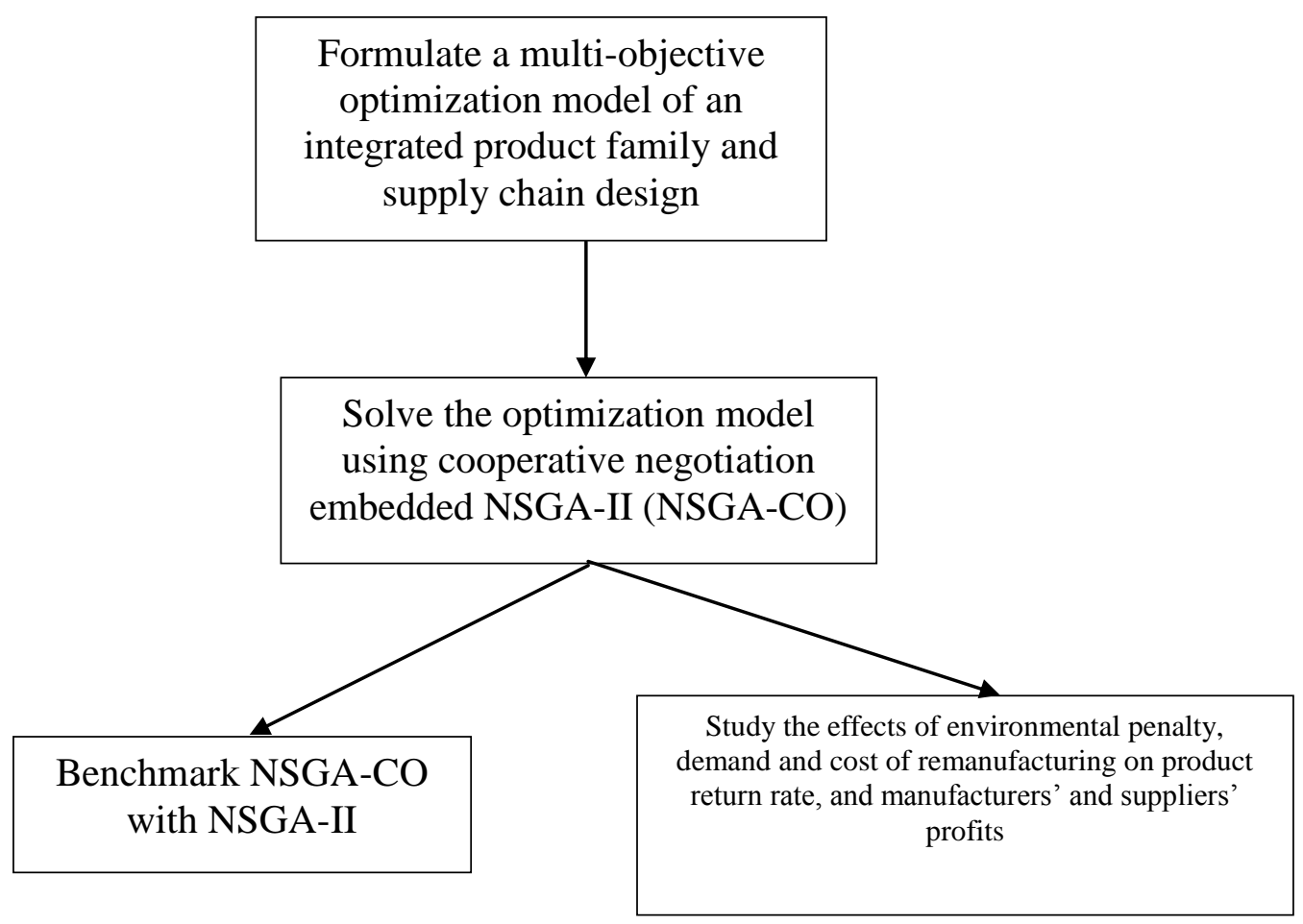

